Citation details:

Liu, Y. (2015) Sound transmission through triple-panel structures lined

with poroelastic materials. J. Sound Vib. 339, pp. 376-395.

doi: 10.1016/j.jsv.2014.11.014

\title{
Sound transmission through triple-panel structures lined with poroelastic materials
}

\author{
$\mathrm{Yu} \mathrm{Liu}{ }^{\mathrm{a}, *}$ \\ ${ }^{a}$ Department of Mechanical Engineering Sciences, University of Surrey, \\ Guildford GU2 7XH, UK
}

\begin{abstract}
In this paper, previous theories on the prediction of sound transmission loss for a double-panel structure lined with poroelastic materials are extended to address the problem of a triple-panel structure. Six typical configurations are considered for a triple-panel structure based on the method of coupling the porous layers to the facing panels which determines critically the sound insulation performance of the system. The transfer matrix method is employed to solve the system by applying appropriate types of boundary conditions for these configurations. The transmission loss of the triple-panel structures in a diffuse sound field is calculated as a function of frequency and compared with that of corresponding double-panel structures. Generally, the triple-panel structure with poroelastic linings has superior acoustic performance to the double-panel counterpart, remarkably in the mid-high frequency range and possibly at low frequencies, by selecting appropriate configurations in which those with two air gaps in the structure exhibit the best overall performance over the entire frequency range. The poroelastic
\end{abstract}

\footnotetext{
*Corresponding author. Tel.: +44 1483682352.

Email address: yu.liu.mes@surrey.ac.uk (Yu Liu)
} 
lining significantly lowers the cut-on frequency above which the triple-panel structure exhibits remarkably higher transmission loss. Compared with a double-panel structure, the wider range of system parameters for a triple-panel structure due to the additional partition provides more design space for tuning the sound insulation performance. Despite the increased structural complexity, the triple-panel structure lined with poroelastic materials has the obvious advantages over its double-panel counterpart while without the penalties in weight and volume, and is hence recommended as a promising replacement for the widely used double-panel sandwich structure in order to optimise the sound transmission loss.

Keywords: Triple-panel structure, Sound transmission loss, Double-panel sandwich structure, Sound insulation performance, Poroelastic material, Biot's theory

\section{Introduction}

2 Double-panel sandwich structures lined with poroelastic materials are 3 widely used for sound insulation purposes in a variety of engineering 4 applications, e.g. automobile, aircraft, and construction industries, 5 due to their superior performance over a wide frequency range and 6 excellent mechanical properties (e.g. stiffness-to-weight ratio, vibration 7 damping) compared with a single panel and other sandwich cores (e.g. 8 air cavity [1-3], corrugated core [4, 5], rib-stiffened core [6-8]). The problem of sound transmission through such a lined panel structure has been studied extensively in literature. Most of these theoretical and experimental studies [9-11] have been restricted to normally incident sound 
to double-panel structures of infinite lateral extent, though the random incidence transmission loss was considered for double-panels without porous linings $[12,13]$. Bolton et al. [14] developed a theoretical model for the prediction of sound transmission through laterally infinite double-panel structures lined with poroelastic materials in a diffuse field. They adapted Biot's theory [15] in this model to describe wave propagation in two-phase poroelastic media and have validated the model with experimental results. A transfer matrix equation was derived by Bolton et al. [14] to efficiently relate the incident waves to and transmitted waves from the multiple panel system. Numerical studies were also conducted for this problem. For example, Panneton and Atalla [16] and Sgard et al. [17] used finite element method and boundary element method to calculate the normal and random incidence transmission losses through finite double panels with porous linings, respectively.

In order to maximise sound transmission loss while minimising total structure mass, double-panel structures with multilayered linings have been applied in many situations. The design of such multilayered panels generally involves a multi-objective optimisation problem [18-20] with regard to material selection, optimal layer thickness and sequencing, and the application of the transfer matrix method to assist the acoustic analysis. Lee et al. [19] used a topology optimisation method for sound transmission through one-dimensional (i.e. normal incidence) multilayered acoustic foams.

They applied open surface conditions to the layer interfaces by assuming insignificant difference between bonded and unbonded multilayers, which is contradictory to the finding by other researchers that the coupling method 
of a double-panel sandwich structure is critical to the sound insulation properties [14, 21]. Tanneau et al. [18] employed a genetic algorithm to solve the optimisation problem for multilayered panels including a combination of solid, fluid, and porous components. However, their model did not consider the case of thin elastic panels in bending [14, 22] whose vibration under the excitation of the stresses exerted by adjacent layers certainly influences the sound insulation of the entire system.

A simple multilayer structure can be obtained by adding an extra middle panel between the two facing panels of a double-panel structure. The resulting triple-panel structure has even better mechanical and thermal insulation properties than its double-panel counterpart, and the introduction of an additional partition that consists of panel, porous material and air gap components provides more options for tuning the sound insulation performance. Despite the increased structural complexity, the triple-panel structure has the potential to be a promising substitute for the double-panel structure widely used in practice as sound insulation devices. However, surprisingly very little work has been reported on acoustic insulation properties of this structure, compared with the research efforts into its double-panel counterpart. The most likely reason is the so-called 'triple leaf effect' observed for windows and walls that makes this structure unpopular in construction industry. It is well known that a triple-glazing window or a triple-leaf wall without sound absorbent linings exhibits inferior low-frequency soundproof performance to its double-leaf counterpart with the same total weight and total air gap depth [23]. This is because the narrower air gaps and lighter panels in a triple-panel structure drive up the 
fundamental resonance frequency of the system. Only above a very high cut-on frequency, $f_{c o}=c / 2 d$ where $c$ is the speed of sound and $d$ is the total air gap depth, is it possible to achieve a noticeably higher transmission loss with a triple-panel wall [24, 25].

The experiments by Tadeu and Mateus [24] have shown that the triple glazing gains reasonable improvements over double glazing yet with penalties in mass and volume. Xin and $\mathrm{Lu}$ [26] developed an analytical model to predict sound transmission of a clamp mounted triple-panel partition and found that the soundproof performance of a triple-panel partition improves as panel dimensions decrease. This model was validated by the experimental results of Brekke [25] but did not consider the effect of poroelastic linings. Sharp [27] proposed an empirical model for the transmission loss of a triple-panel wall with absorption equivalent to at least 2 inches of fiberglass batts in each cavity, and Jones [28] suggested improvements to this model by correcting several errors in the original theory by Sharp [27]. Moreover, controversial results exist regarding the performance of a triple-panel wall below the fundamental resonance frequency $f_{m}$. Sharp $[27,29]$ reported the same transmission loss below $f_{m}$ for both double- and triple-wall structures, whereas Quirt [30] measured a slightly larger transmission loss below $f_{m}$ for a triple-glazing window as predicted by Brekke [25].

Sound absorbing porous linings will enhance the sound insulation properties of a multiple-panel structure. It is therefore of interest to explore how a triple-panel structure lined with poroelastic materials will improve in sound insulation properties over its double-panel counterpart, for instance higher transmission loss at lower frequencies. This problem has not been 
addressed yet to the best of the author's knowledge and will be a focus of the present study. The prime objective of this paper is three-fold: (i) to extend the theoretical model of Bolton et al. [14] to consider the case of a triple-panel structure by modifying the boundary conditions and the transfer matrix; (ii) to predict the transmission loss of various configurations of the triple-panel structure and examine the sound insulation properties by comparing with the double-panel counterparts; and (iii) to explore the options of sound insulation control for the triple-panel structure through tuning some typical parameters (e.g. thicknesses of porous layers and air gaps, air properties in the gaps) and the potential advantages over the double-panel structure.

The remaining paper is organised as follows. In Section 2 the theoretical framework for sound transmission through a triple-panel structure with poroelastic linings is established on the basis of Bolton et al.'s model [14] and Biot's theory [15], including the boundary conditions, transfer matrix method and random incidence transmission loss. The results of transmission loss, effects of typical parameters for control, and comparison between the double- and triple-panel structures are presented and discussed in Section 3. The conclusions are summarised in the last section along with suggestions for future work.

\section{Theoretical formulation}

\subsection{General description of the system}

The triple-panel structure considered in this study consists of three parallel homogeneous thin panels, poroelastic material linings and air gaps (if any) between the panels, as illustrated in Fig. 1. The layers of the panel, 


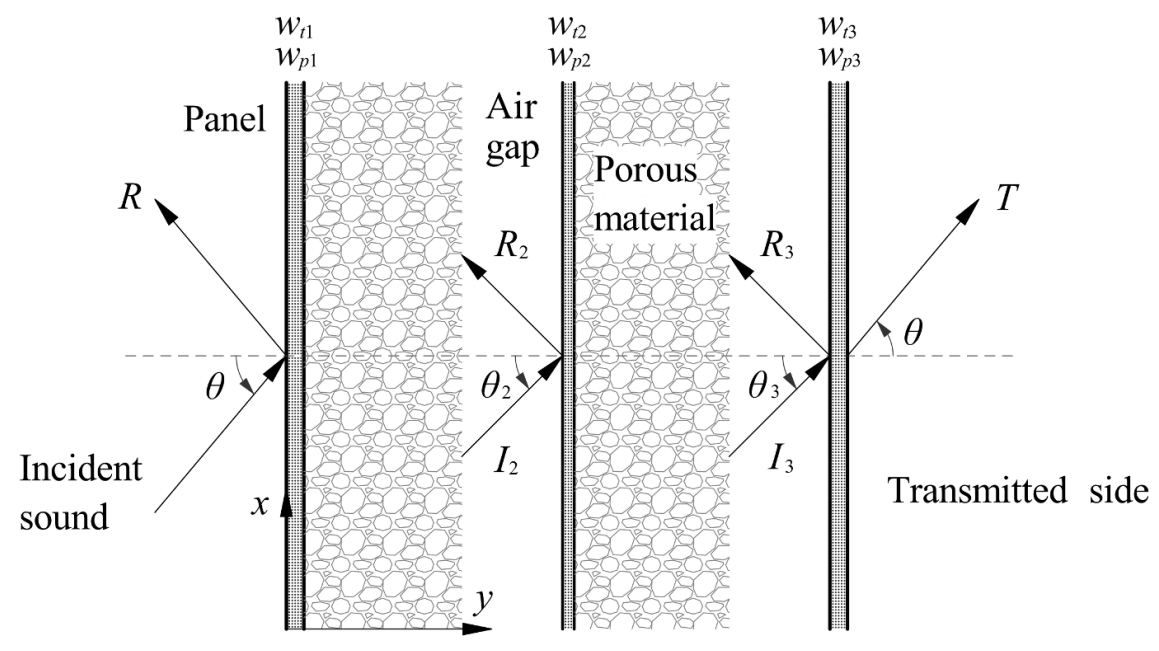

Figure 1: Schematic of a plane wave transmitting through a triple-panel structure.

poroelastic material and air gap have finite thicknesses and are assumed to be infinite in lateral extent. The structure is situated in ambient air which is considered to be semi-infinite before the first panel and behind the third panel. A plane sound wave of unit amplitude is incident on the first panel and transmits through the system in a direction parallel with the $x-y$ plane. Following Bolton et al. [14], the problem is formulated in a two-dimensional form and the velocity potential of the incident wave can be expressed in a harmonic form as

$$
\phi_{i}=\mathrm{e}^{\mathrm{j} \omega t-\mathrm{j}\left(k_{x} x+k_{y} y\right)},
$$

where the wavenumber $k=\omega / c, \omega$ is angular frequency, $c$ is the ambient speed of sound, and the symbol $\mathrm{j}=\sqrt{-1}$; the components of $k$ are

$$
k_{x}=k \sin \theta \quad \text { and } \quad k_{y}=k \cos \theta
$$

with $\theta$ the angle of incidence. On the incident side of the system, the sound field consists of incident and reflected plane waves, and the velocity potential 
is written as

$$
\phi_{1}=\phi_{i}+\phi_{r}=\mathrm{e}^{\mathrm{j} \omega t-\mathrm{j} k_{x} x}\left(\mathrm{e}^{-\mathrm{j} k_{y} y}+R \mathrm{e}^{\mathrm{j} k_{y} y}\right),
$$

where $\phi_{r}$ is the velocity potential of the reflected wave and $R$ is the amplitude of $\phi_{r}$. On the transmitted side, an anechoic termination allows only a single plane transmitted wave present, and similarly its velocity potential can be written as

$$
\phi_{t}=T \mathrm{e}^{\mathrm{j} \omega t-\mathrm{j}\left(k_{x} x+k_{y} y\right)},
$$

where $T$ is the amplitude of $\phi_{t}$ and also the transmission coefficient.

The velocity potentials in the two air gaps between the panels are

$$
\begin{aligned}
& \phi_{2}=\phi_{2 i}+\phi_{2 r}=\mathrm{e}^{\mathrm{j} \omega t-\mathrm{j} k_{x} x}\left(I_{2} \mathrm{e}^{-\mathrm{j} k_{2 y} y}+R_{2} \mathrm{e}^{\mathrm{j} k_{2 y} y}\right), \\
& \phi_{3}=\phi_{3 i}+\phi_{3 r}=\mathrm{e}^{\mathrm{j} \omega t-\mathrm{j} k_{x} x}\left(I_{3} \mathrm{e}^{-\mathrm{j} k_{3 y} y}+R_{3} \mathrm{e}^{\mathrm{j} k_{3 y} y}\right),
\end{aligned}
$$

respectively, where the coefficients $I_{2}, R_{2}, I_{3}, R_{3}$ are the amplitudes of the incident and reflected wave components within the two air gaps. The air properties in the two gaps, i.e. $\rho_{2}, c_{2}, \rho_{3}, c_{3}$, can be different from those of the ambient air $\left(\rho_{0}, c\right)$, and so the wavenumbers in the air gaps are defined as $k_{2}=\omega / c_{2}$ and $k_{3}=\omega / c_{3}$. The $y$-components of $k_{2}$ and $k_{3}$ are

$$
k_{2 y}=k_{2} \cos \theta_{2} \quad \text { and } \quad k_{3 y}=k_{3} \cos \theta_{3},
$$

where $\theta_{2}$ and $\theta_{3}$ are the respective incidence angles of the waves transmitting in the two air gaps. According to the well-known Snell-Descartes law, the wavenumbers and incidence angles of waves $m$ and $n$ transmitting in two adjacent media are related through

$$
k_{m} \sin \theta_{m}=k_{n} \sin \theta_{n},
$$


154

and hence all wave components in the different layers of the system must share the same trace wavenumber $k_{x}[31]$.

Finally, the transverse displacements of the three panels excited by the incident sound wave can be expressed as [14]

$$
w_{t i}=W_{t i} \mathrm{e}^{\mathrm{j} \omega t-\mathrm{j} k_{x} x}, \quad i=1,2,3
$$

where $W_{t i}$ are the respective amplitudes of the three panels' transverse displacement. Similarly, the centre-line, in-plane displacements of the three panels are given by

$$
w_{p i}=W_{p i} \mathrm{e}^{\mathrm{j} \omega t-\mathrm{j} k_{x} x}, \quad i=1,2,3
$$

with $W_{p i}$ being the corresponding amplitudes of $w_{p i}$.

\subsection{Biot's theory of waves in porous medium}

The theory developed by Biot [15] to describe wave propagation in poroelastic materials is summarised in this section. A more detailed description of the model can be found in Refs. [14, 15, 21, 22]. According to Biot's theory, a poroelastic material is modelled as a coupled system consisting of a solid and a fluid phase with three waves transmitting in both phases: two longitudinal waves and a transverse wave. The governing equations for the propagation of the three waves are

$$
\nabla^{4} e+A_{1} \nabla^{2} e+A_{2} e=0
$$

$$
\nabla^{2} \boldsymbol{\omega}+k_{r}^{2} \boldsymbol{\omega}=0
$$

where $e=\nabla \cdot \mathbf{u}$ and $\mathbf{\omega}=\nabla \times \mathbf{u}$ denote the volumetric and rotational strains of the solid phase respectively, $\mathbf{u}$ being the displacement vector of the 
solid phase. The volumetric and rotational strains of the fluid phase can be obtained via

$$
\begin{aligned}
\epsilon & =\nabla \cdot \mathbf{U}=\frac{\left(P R-Q^{2}\right) \nabla^{2} e+\omega^{2}\left(\rho_{11}^{*} R-\rho_{12}^{*} Q\right) e}{\omega^{2}\left(\rho_{22}^{*} Q-\rho_{12}^{*} R\right)} \\
\boldsymbol{\Omega} & =\nabla \times \mathbf{U}=-\frac{\rho_{12}^{*}}{\rho_{22}^{*}} \boldsymbol{\omega}
\end{aligned}
$$

once the solutions of $e$ and $\boldsymbol{\omega}$ are known, where $\mathbf{U}$ is the fluid displacement vector. Since Eq. (10) is a fourth-order wave equation, two solutions can exist, i.e. two dilatational wave types with the wavenumbers given by

$$
k_{\mathrm{I}, \mathrm{II}}^{2}=\left(A_{1} \pm \sqrt{A_{1}^{2}-4 A_{2}}\right) / 2 .
$$

The second-order wave equation (11) indicates the existence of only a single rotational wave with the wavenumber $k_{r}$ given by

$$
k_{r}^{2}=\left(\omega^{2} / N\right)\left(\rho_{11}^{*}-\rho_{12}^{* 2} / \rho_{22}^{*}\right) .
$$

164 In the governing equation (10), the coefficients $A_{1}$ and $A_{2}$ are written as

$$
\begin{aligned}
& A_{1}=\omega^{2}\left(\rho_{11}^{*} R^{\prime}-2 \rho_{12}^{*} Q+\rho_{22}^{*} P\right) /\left(P R^{\prime}-Q^{2}\right), \\
& A_{2}=\omega^{4}\left(\rho_{11}^{*} \rho_{22}^{*}-\rho_{12}^{* 2}\right) /\left(P R^{\prime}-Q^{2}\right) .
\end{aligned}
$$

In the above expression, $P=A+2 N$ with

$$
A=\frac{\nu E_{1}}{(1+\nu)(1-2 \nu)}, \quad N=\frac{E_{1}}{2(1+\nu)},
$$

where $N$ denotes the elastic shear modulus and $A$ the first Lamé constant; $E_{1}$ and $\nu$ are the Young's modulus and Poisson's ratio of the solid phase. $E_{1}$ is complex valued: $E_{1}=E_{m} /(1+\mathrm{j} \eta)$, where $E_{m}$ is the static Young's modulus and $\eta$ is the loss factor. The coefficients $Q$ and $R^{\prime}$ represent the 
elastic coupling between the volume change of the solid phase and that of the interstitial fluid,

$$
Q=(1-h) E_{2}, \quad R^{\prime}=h E_{2},
$$

where $E_{2}$ denotes the bulk modulus of the fluid in pores and $h$ is the porosity of the porous material. In this model, the interstitial fluid is assumed to be air and it shares the same air properties (i.e. $\rho_{f}, c_{f}$ ) as those of the adjacent air gap (if any). Assuming cylindrical pores, $E_{2}$ is related to the bulk modulus of air as $[14,32,33]$

$$
\begin{aligned}
E_{2} & =E_{0}\left[1+2(\gamma-1) / \lambda \cdot T_{c}(\lambda)\right]^{-1}, \\
T_{c}(\lambda) & =J_{1}(\lambda) / J_{0}(\lambda), \\
\lambda & =\operatorname{Pr}^{1 / 2} \lambda_{c} \sqrt{-\mathrm{j}}, \quad \lambda_{c}^{2}=8 \omega \rho_{f} \epsilon^{\prime} / h \sigma,
\end{aligned}
$$

where $E_{0}=\rho_{f} c_{f}^{2}, \gamma$ is the ratio of specific heats, $J_{i}$ is the first-kind Bessel function of order $i, \operatorname{Pr}$ is the Prandtl number, $\epsilon^{\prime}$ is the geometrical structure factor [34] and $\sigma$ is the steady-state, macroscopic flow resistivity.

The complex equivalent densities in Eq. (15) are defined as

$$
\begin{array}{ll}
\rho_{11}^{*}=\rho_{11}+b / \mathrm{j} \omega, & \rho_{11}=\rho_{1}+\rho_{a}, \\
\rho_{12}^{*}=\rho_{12}-b / \mathrm{j} \omega, & \rho_{12}=-\rho_{a}, \\
\rho_{22}^{*}=\rho_{22}+b / \mathrm{j} \omega, & \rho_{22}=\rho_{2}+\rho_{a},
\end{array}
$$

where $\rho_{1}$ and $\rho_{2}=\rho_{f} h$ are the bulk densities of the solid and fluid phases of porous materials, respectively; $\rho_{a}=\rho_{2}\left(\epsilon^{\prime}-1\right)$ is an inertial coupling parameter between the fluid and solid $[22,35]$, and $\rho_{11}, \rho_{22}$ represent the effective densities of the solid and fluid phases [14], respectively. The 
coefficient $b$ is a viscous coupling factor expressed as $[22,35]$

$$
b=\mathrm{j} \omega \epsilon^{\prime} \rho_{2}\left(\rho_{c}^{*} / \rho_{f}-1\right),
$$

186 where the complex density

$$
\rho_{c}^{*}=\rho_{f}\left[1-2 / \lambda_{c} \sqrt{-\mathrm{j}} \cdot T_{c}\left(\lambda_{c} \sqrt{-\mathrm{j}}\right)\right]^{-1}
$$

187 for cylindrical pores $[11,32]$.

The solutions of $e, \boldsymbol{\omega}, \epsilon, \boldsymbol{\Omega}$ have been derived in previous studies [14, 22]. From these solutions the displacement components for the solid and fluid phases can be obtained as [14]

$$
\begin{aligned}
u_{x} & =\mathrm{j} k_{x} \mathrm{e}^{\mathrm{j} \omega t-\mathrm{j} k_{x} x}\left(\frac{C_{1}}{k_{\mathrm{I}}^{2}} \mathrm{e}^{-\mathrm{j} k_{\mathrm{I} y} y}+\frac{C_{2}}{k_{\mathrm{I}}^{2}} \mathrm{e}^{\mathrm{j} k_{\mathrm{I} y} y}+\frac{C_{3}}{k_{\mathrm{II}}^{2}} \mathrm{e}^{-\mathrm{j} k_{\mathrm{II} y} y}+\frac{C_{4}}{k_{\mathrm{II}}^{2}} \mathrm{e}^{\mathrm{j} k_{\mathrm{II} y} y}\right) \\
& -\mathrm{j} \frac{k_{r y}}{k_{r}^{2}} \mathrm{e}^{\mathrm{j} \omega t-\mathrm{j} k_{x} x}\left(C_{5} \mathrm{e}^{-\mathrm{j} k_{r y} y}-C_{6} \mathrm{e}^{\mathrm{j} k_{r y} y}\right), \\
u_{y} & =\mathrm{j} \mathrm{e}^{\mathrm{j} \omega t-\mathrm{j} k_{x} x}\left(\frac{k_{\mathrm{I} y}}{k_{\mathrm{I}}^{2}} C_{1} \mathrm{e}^{-\mathrm{j} k_{\mathrm{I} y} y}-\frac{k_{\mathrm{I} y}}{k_{\mathrm{I}}^{2}} C_{2} \mathrm{e}^{\mathrm{j} k_{\mathrm{I} y} y}+\frac{k_{\mathrm{II} y}}{k_{\mathrm{II}}^{2}} C_{3} \mathrm{e}^{-\mathrm{j} k_{\mathrm{II} y} y}-\frac{k_{\mathrm{II} y}}{k_{\mathrm{II}}^{2}} C_{4} \mathrm{e}^{\mathrm{j} k_{\mathrm{II} y} y}\right) \\
& +\mathrm{j} \frac{k_{x}}{k_{r}^{2}} \mathrm{e}^{\mathrm{j} \omega t-\mathrm{j} k_{x} x}\left(C_{5} \mathrm{e}^{-\mathrm{j} k_{r y} y}+C_{6} \mathrm{e}^{\mathrm{j} k_{r y} y}\right), \\
U_{x} & =\mathrm{j} k_{x} \mathrm{e}^{\mathrm{j} \omega t-\mathrm{j} k_{x} x}\left(b_{1} \frac{C_{1}}{k_{\mathrm{I}}^{2}} \mathrm{e}^{-\mathrm{j} k_{\mathrm{I} y} y}+b_{1} \frac{C_{2}}{k_{\mathrm{I}}^{2}} \mathrm{e}^{\mathrm{j} k_{\mathrm{I} y} y}+b_{2} \frac{C_{3}}{k_{\mathrm{II}}^{2}} \mathrm{e}^{-\mathrm{j} k_{\mathrm{II} y} y}+b_{2} \frac{C_{4}}{k_{\mathrm{II}}^{2}} \mathrm{e}^{\mathrm{j} k_{\mathrm{II} y} y}\right) \\
& -\mathrm{j} g \frac{k_{r y}}{k_{r}^{2}} \mathrm{e}^{\mathrm{j} \omega t-\mathrm{j} k_{x} x}\left(C_{5} \mathrm{e}^{-\mathrm{j} k_{r y} y}-C_{6} \mathrm{e}^{\mathrm{j} k_{r y} y}\right), \\
U_{y} & =\mathrm{j} \mathrm{e}^{\mathrm{j} \omega t-\mathrm{j} k_{x} x}\left(b_{1} \frac{k_{\mathrm{I} y}}{k_{\mathrm{I}}^{2}} C_{1} \mathrm{e}^{-\mathrm{j} k_{\mathrm{I} y} y}-b_{1} \frac{k_{\mathrm{I} y}}{k_{\mathrm{I}}^{2}} C_{2} \mathrm{e}^{\mathrm{j} k_{\mathrm{I} y} y}+b_{2} \frac{k_{\mathrm{II} y}}{k_{\mathrm{II}}^{2}} C_{3} \mathrm{e}^{-\mathrm{j} k_{\mathrm{II} y} y}-b_{2} \frac{k_{\mathrm{II} y}}{k_{\mathrm{II}}^{2}} C_{4} \mathrm{e}^{\mathrm{j} k_{\mathrm{II} y} y}\right) \\
& +\mathrm{j} g \frac{k_{x}}{k_{r}^{2}} \mathrm{e}^{\mathrm{j} \omega t-\mathrm{j} k_{x} x}\left(C_{5} \mathrm{e}^{-\mathrm{j} k_{r y} y}+C_{6} \mathrm{e}^{\mathrm{j} k_{r y} y}\right),
\end{aligned}
$$

188 where

$$
\begin{aligned}
& b_{1}=a_{1}-a_{2} k_{\mathrm{I}}^{2}, \quad b_{2}=a_{1}-a_{2} k_{\mathrm{II}}^{2}, \\
& a_{1}=\left(\rho_{11}^{*} R^{\prime}-\rho_{12}^{*} Q\right) /\left(\rho_{22}^{*} Q-\rho_{12}^{*} R^{\prime}\right), \\
& a_{2}=\left(P R^{\prime}-Q^{2}\right) /\left[\omega^{2}\left(\rho_{22}^{*} Q-\rho_{12}^{*} R^{\prime}\right)\right],
\end{aligned}
$$


and

$$
k_{\mathrm{I}, \mathrm{II} y}^{2}=k_{\mathrm{I}, \mathrm{II}}^{2}-k_{x}^{2}, \quad g=-\rho_{12}^{*} / \rho_{22}^{*} .
$$

The constants $C_{1}-C_{6}$ represent the complex amplitudes of six wave components, i.e. positive- and negative-propagating components of the two longitudinal waves and one transverse wave, and will be determined by applying appropriate boundary conditions as discussed in Section 2.3.

The stresses in the two phases of poroelastic medium can be obtained from the solutions of $e$ and $\epsilon$. Here only the normal solid stress in the $y$-direction $\sigma_{y}$, the fluid stress $s$, and the solid shear stress in the $x-y$ plane $\tau_{x y}$ are discussed which will be used in the boundary conditions. These stresses are determined through the relations:

$$
\begin{aligned}
\sigma_{y} & =2 N e_{y}+A e+Q \epsilon, \quad s=R^{\prime} \epsilon+Q e, \\
\tau_{x y} & =\tau_{y x}=N\left(\partial u_{x} / \partial y+\partial u_{y} / x\right)
\end{aligned}
$$

and their expressions are in the form [14]:

$$
\begin{aligned}
\sigma_{y}=\mathrm{e}^{\mathrm{j} \omega t-\mathrm{j} k_{x} x} & {\left[\left(2 N \frac{k_{\mathrm{I} y}^{2}}{k_{\mathrm{I}}^{2}}+A+b_{1} Q\right)\left(C_{1} \mathrm{e}^{-\mathrm{j} k_{\mathrm{I} y} y}+C_{2} \mathrm{e}^{\mathrm{j} k_{\mathrm{I} y} y}\right)\right.} \\
+ & \left(2 N \frac{k_{\mathrm{II} y}^{2}}{k_{\mathrm{II}}^{2}}+A+b_{2} Q\right)\left(C_{3} \mathrm{e}^{-\mathrm{j} k_{\mathrm{II} y} y}+C_{4} \mathrm{e}^{\mathrm{j} k_{\mathrm{II} y} y}\right) \\
+ & \left.2 N \frac{k_{x} k_{r y}}{k_{r}^{2}}\left(C_{5} \mathrm{e}^{-\mathrm{j} k_{r y} y}-C_{6} \mathrm{e}^{\mathrm{j} k_{r y} y}\right)\right], \\
s=\mathrm{e}^{\mathrm{j} \omega t-\mathrm{j} k_{x} x} & {\left[\left(Q+b_{1} R^{\prime}\right)\left(C_{1} \mathrm{e}^{-\mathrm{j} k_{\mathrm{II} y} y}+C_{2} \mathrm{e}^{\mathrm{j} k_{I y} y}\right)\right.} \\
+ & \left.\left(Q+b_{2} R^{\prime}\right)\left(C_{3} \mathrm{e}^{-\mathrm{j} k_{\mathrm{II} y} y}+C_{4} \mathrm{e}^{\mathrm{j} k_{\mathrm{II} y} y}\right)\right], \\
\tau_{x y}=\mathrm{e}^{\mathrm{j} \omega t-\mathrm{j} k_{x} x} N & {\left[\frac{2 k_{x} k_{\mathrm{I} y}}{k_{\mathrm{I}}^{2}}\left(C_{1} \mathrm{e}^{-\mathrm{j} k_{\mathrm{I} y} y}-C_{2} \mathrm{e}^{\mathrm{j} k_{\mathrm{I} y} y}\right)+\frac{2 k_{x} k_{\mathrm{II} y}}{k_{\mathrm{II}}^{2}}\left(C_{3} \mathrm{e}^{-\mathrm{j} k_{\mathrm{II} y} y}-C_{4} \mathrm{e}^{\mathrm{j} k_{\mathrm{II} y} y}\right)\right.} \\
+ & \left.\frac{k_{x}^{2}-k_{r y}^{2}}{k_{r}^{2}}\left(C_{5} \mathrm{e}^{-\mathrm{j} k_{r y} y}+C_{6} \mathrm{e}^{\mathrm{j} k_{r y} y}\right)\right] .
\end{aligned}
$$




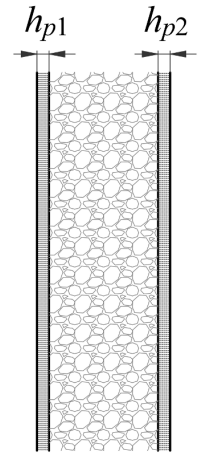

$\mathrm{BB}$

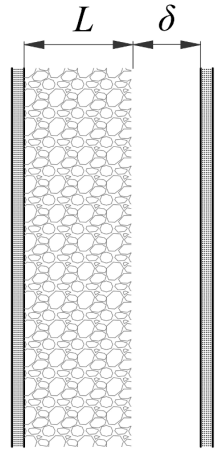

BU

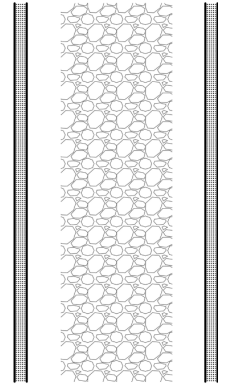

$\mathrm{UU}$

Figure 2: The three configurations of a double-panel structure considered in Ref. [14].

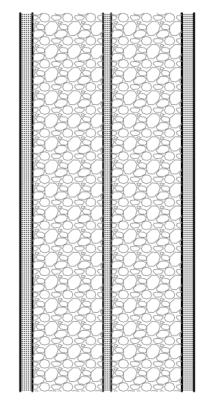

BBBB

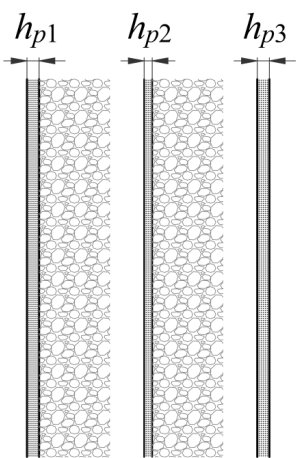

BUBU

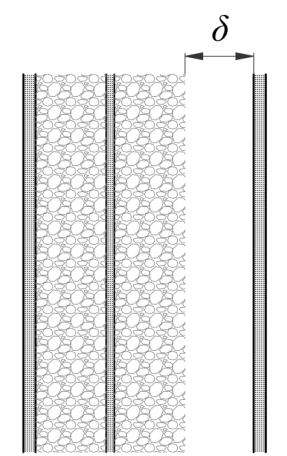

BBBU

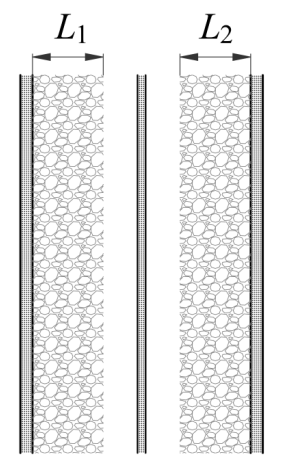

BUUB

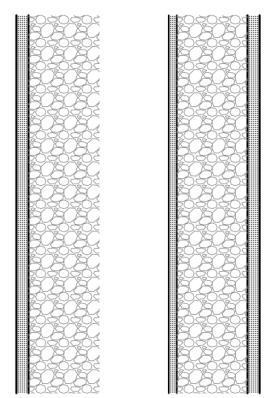

BUBB

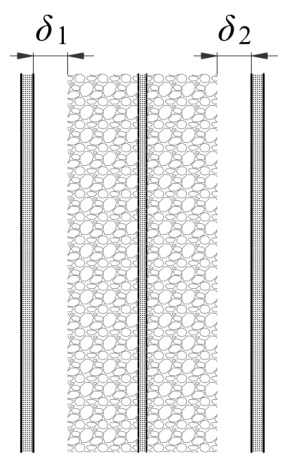

UBBU

Figure 3: Six typical configurations of a triple-panel structure considered in this study. 


\subsection{Triple-panel configurations and boundary conditions}

Bolton et al. [14] considered two basic methods of coupling a porous layer to an elastic panel, i.e., 1) "bonded" (B): the porous layer is bonded directly to the facing panel, and 2) "unbonded" (U): the porous layer is separated from the panel by an air gap. Based on these two coupling methods, they studied three different cases, as shown in Fig. 2, for a double-panel structure: 1) a bonded-bonded (BB) configuration where the porous foam is bonded to the two panels on both sides, 2) a bonded-unbonded (BU) configuration where the porous foam is bonded to the first panel on one side whereas unbonded to the second panel on the other side, and 3) an unbonded-unbonded (UU) configuration where the porous foam is unbonded to the two panels on both sides.

In the case of a triple-panel structure, the introduction of an extra partition comprised of panel, porous material and air gap (where applicable) leads to the coupling of two porous layers and three panels and hence more configurations than its double-panel counterpart. Figure 3 illustrates six typical configurations for a triple-panel structure considered in this study which are denoted as BBBB, BBBU, BUBB, BUBU, BUUB and UBBU, respectively. According to the number of air gaps between the panel and porous layer (i.e. the number of $U$ coupling methods), these different configurations can be divided into three categories, i.e.,

- Category (i): no air gap (BBBB),

- Category (ii): a single air gap (BBBU and BUBB),

- Category (iii): two air gaps (BUBU, BUUB and UBBU). 
As will be shown in Section 3.3, the soundproof performance of a triple-panel configuration depends, to a great extent, on the number of air gaps existing in the structure and behaves distinctly across the three categories.

Appropriate boundary conditions $(\mathrm{BC})$ are needed to determine the constants $C_{1}-C_{6}$ in Eqs. (22) and (26). These constants of wave amplitudes in the porous material will be doubled (i.e. $C_{1}-C_{12}$ ) for the case of a triple-panel configuration due to the extra porous layer. Bolton et al. [14] proposed three types of BC for a double-panel structure at the surfaces of a porous layer and a panel, which also apply to a triple-panel structure and will be introduced respectively. A fourth type of $\mathrm{BC}$ required for a triple-panel structure will also be defined. The first type of boundary conditions, BC (I), applies to the open surface of a porous layer and is related to any configurations that include an unbonded (U) coupling between the foam and panel (e.g. BU, BUBB, BUBU). The four conditions for BC (I) to be satisfied are, respectively:

$$
\begin{gathered}
\text { (i) }-h p=s, \quad(\text { ii })-(1-h) p=\sigma_{y}, \\
\text { (iii) } v_{y}=(1-h) \frac{\partial u_{y}}{\partial t}+h \frac{\partial U_{y}}{\partial t}, \quad \text { (iv) } \tau_{x y}=0,
\end{gathered}
$$

where $p$ and $v_{y}$ are the acoustic pressure and the normal component of the acoustic particle velocity in the exterior medium at the interface and can be simply obtained from the velocity potential $\phi$ of the sound waves propagating in the exterior medium, i.e.

$$
p=\rho \frac{\partial \phi}{\partial t}=\mathrm{j} \omega \rho \phi, \quad v_{y}=-\frac{\partial \phi}{\partial y},
$$

$\rho$ being the density of the exterior fluid. The first two conditions relate the acoustic pressure in the exterior medium to the normal stresses acting on the fluid and solid phases of the poroelastic material, respectively. The 
third condition is the continuity relation of normal velocity between the exterior and porous media. The last condition represents no shear stress at the interface because an inviscid exterior fluid is assumed.

The second type of boundary conditions, BC (II), applies to a panel bonded to poroelastic material on one side and open to the exterior medium on the other side, for example, both panels in BB, the first panel in BBBU, and the first two panels in BUBB and BUBU, as shown in Figs. 2 and 3. Six conditions must be satisfied for BC (II):

$$
\begin{aligned}
& \text { (i) } v_{y}=\frac{\partial w_{t}}{\partial t}, \quad\left(\text { ii) } u_{y}=w_{t}, \quad(\text { iii }) U_{y}=w_{t},\right. \\
& \text { (iv) } u_{x}=w_{p} \mp \frac{h_{p}}{2} \frac{\partial w_{t}}{\partial x} \\
& \text { (v) } \pm \tau_{y x}=\left(D_{p} k_{x}^{2}-\omega^{2} m_{s}\right) w_{p}, \\
& \text { (vi) } \pm p \mp q_{p}-\mathrm{j} k_{x} \frac{h_{p}}{2} \tau_{x y}=\left(D k_{x}^{4}-\omega^{2} m_{s}\right) w_{t},
\end{aligned}
$$

where $h_{p}$ denotes the panel thickness, $D$ and $D_{p}$ the flexural and longitudinal stiffness per unit width of the panel respectively, $m_{s}$ the panel mass per unit area, and $q_{p}=-\sigma_{y}-s$ the normal stress exerted by the poroelastic material on the panel. The first three conditions represent the continuity of normal velocity and normal displacement at the interface of the porous material and panel. The fourth condition equates the tangential displacement of the porous material's solid phase to the centre-line in-plane displacement of the panel vibrating in flexure [36]. The conditions (v) and (vi) represent the panel in-plane and transverse accelerations under the excitation of the shear and normal stresses, respectively, where Eq. (29vi) is the Euler-Bernoulli transverse panel equation with the distributed moment due to the in-plane shear force taken into account. In BC (II) (iv)-(vi), the first and second 
signs are taken for the porous material attached to the panel on the positive and negative $y$-facing surfaces, respectively.

The third type of boundary conditions, BC (III), applies to a panel unbonded to a porous layer on both sides, for example, the two panels in UU, the last panel in BBBU and BUBU, and the middle panel in BUUB (see Figs. 2 and 3). Three boundary conditions should be satisfied for BC (III) at the centre line of the panel:

$$
\begin{aligned}
& \text { (i) } v_{1 y}=\frac{\partial w_{t}}{\partial t}, \quad \text { (ii) } v_{2 y}=\frac{\partial w_{t}}{\partial t}, \\
& \text { (iii) } p_{1}-p_{2}=\left(D k_{x}^{4}-\omega^{2} m_{s}\right) w_{t},
\end{aligned}
$$

where $p_{1,2}$ and $v_{1,2 y}$ are the acoustic pressures and normal acoustic particle velocities at the two surfaces of the panel.

For a triple-panel structure, the presence of a middle panel results in a fourth type of boundary conditions, BC (IV). This type of boundary conditions applies to a middle panel bonded to poroelastic materials on both sides, and is required for the configurations in Fig. 3 using the BB coupling at the middle panel, i.e. BBBB, BBBU and UBBU. Similar to BC (II), eight boundary conditions must be satisfied for BC (IV), i.e.

$$
\begin{aligned}
& \text { (i) } u_{y 1}=w_{t}, \quad \text { (ii) } U_{y 1}=w_{t}, \quad(\text { iii }) u_{y 2}=w_{t}, \quad \text { (iv) } U_{y 2}=w_{t}, \\
& \text { (v) } u_{x 1}=w_{p}+\frac{h_{p}}{2} \frac{\partial w_{t}}{\partial x}, \quad(\text { vi }) u_{x 2}=w_{p}-\frac{h_{p}}{2} \frac{\partial w_{t}}{\partial x} \\
& \text { (vii) } \tau_{y x 2}-\tau_{y x 1}=\left(D_{p} k_{x}^{2}-\omega^{2} m_{s}\right) w_{p}, \\
& \text { (vi) } q_{p 1}-q_{p 2}-\mathrm{j} k_{x} \frac{h_{p}}{2}\left(\tau_{x y 1}+\tau_{x y 2}\right)=\left(D k_{x}^{4}-\omega^{2} m_{s}\right) w_{t} .
\end{aligned}
$$

The first four conditions simply require that the normal displacements of the
solid and fluid phases of the two adjacent porous layers are equal to the panel 
transverse displacement. The conditions (v) and (vi) are an application of Eq. (29iv) with the sign taken appropriately for the two porous layers bonded on both surfaces of the middle panel. The seventh condition is a variant of BC (II) (v) where the shear stress $\tau_{y x}$ is updated as a sum of the shear stresses exerted by the two adjacent porous layers. Similarly, the last condition is the variant Euler-Bernoulli transverse panel equation for the BB middle panel which includes the contributions of normal and shear stresses from both porous layers acting on the panel but ignores the acoustic pressure owing to the sealed surfaces of the middle panel without any air gap.

\subsection{Transfer matrix equation and transmission loss}

A transfer matrix equation can be derived by applying the appropriate types of boundary conditions BC (I)-(IV) for a specific configuration and rearranging the equations into a matrix form,

$$
\mathbf{A}_{n} \mathbf{C}_{n}=\mathbf{B}_{n},
$$

where $\mathbf{A}_{n}$ is a $n \times n$ transfer coefficient matrix, $\mathbf{C}_{n}$ is a $n \times 1$ vector of unknown amplitudes, and $\mathbf{B}_{n}$ is a $n \times 1$ forcing vector. The $i$ th row of the matrix equation (32) corresponds to the $i$ th boundary condition, and $n$ is the total number of equations. Substituting the various solutions for $u_{x}, u_{y}, U_{x}, U_{y}, \sigma_{y}, s, \tau_{x y}$ given in Eqs. (22) and (26) into BC (I)-(IV), the resulting transfer matrix equation includes the unknown complex wave amplitudes in the porous materials $\left(C_{1}-C_{12}\right)$, facing panels $\left(W_{t 1,2,3}, W_{p 1,2,3}\right)$, and air gaps $\left(I_{2,3}, R_{2,3}\right.$ where applicable) along with the reflected and transmitted wave amplitudes $(R, T)$ that are needed for calculating the transmission loss of the system. The number of unknown amplitudes in 
Table 1: The vector $\mathbf{C}$ of unknown wave amplitudes for the six typical configurations of a triple-panel structure.

\begin{tabular}{clll}
\hline Configuration & $\mathbf{C}_{n}^{T}$ & $n$ \\
\hline BBBB & {$\left[C_{1}-C_{12} W_{t 1} W_{t 2} W_{t 3} W_{p 1} W_{p 2} W_{p 3} R T\right]$} & 20 \\
BBBU & {$\left[C_{1}-C_{12} I_{3} R_{3} W_{t 1} W_{t 2} W_{t 3} W_{p 1} W_{p 2} R T\right]$} & 21 \\
BUBB & {$\left[C_{1}-C_{12} I_{2} R_{2} W_{t 1} W_{t 2} W_{t 3} W_{p 1} W_{p 2} W_{p 3} R T\right]$} & 22 \\
BUBU & {$\left[C_{1}-C_{12} I_{2} R_{2} I_{3} R_{3} W_{t 1} W_{t 2} W_{t 3} W_{p 1} W_{p 2} R T\right]$} & 23 \\
BUUB & {$\left[C_{1}-C_{12} I_{2} R_{2} I_{3} R_{3} W_{t 1} W_{t 2} W_{t 3} W_{p 1} W_{p 3} R T\right]$} & 23 \\
$\mathrm{UBBU}$ & {$\left[C_{1}-C_{12} I_{2} R_{2} I_{3} R_{3} W_{t 1} W_{t 2} W_{t 3} W_{p 2} R T\right]$} & 22 \\
\hline
\end{tabular}

the vector $\mathbf{C}_{n}$ depends on the particular configuration of the triple-panel structure. The forms of the unknown vector $\mathbf{C}$ are provided in Table 1 for the six typical triple-panel configurations shown in Fig. 3. An example solution for the BBBU configuration can be found in Appendix A which gives the elements of the transfer coefficient matrix $\mathbf{A}$ and the forcing vector $\mathbf{B}$.

By solving the matrix equation (32) for any specific frequencies, one obtains the solution for the complex wave amplitudes in $\mathbf{C}$ including the transmitted wave amplitude $T$. Since in a diffuse sound field random incident waves can be from all directions, the solution of $T$ can be expressed as a function of frequency $\omega$ and incidence angle $\theta$. The power transmission coefficient is defined simply as the square of the modulus of the pressure transmission coefficient, i.e. $\tau=|T|^{2}$ since the incident sound is of unit amplitude. In the case of a diffuse sound field, incident angles are assumed to be uniformly distributed over a range $\left[0, \theta_{m}\right]$, where $\theta_{m}$ is the maximum 
angle above which no incidence sound exists. The average of the power transmission coefficient $\tau$ over all possible angles of incidence is then obtained by integrating over the range of incidence angle [31]:

$$
\bar{\tau}=2 \int_{0}^{\theta_{m}} \tau(\theta) \sin \theta \cos \theta \mathrm{d} \theta .
$$

The maximum incidence angle $\theta_{m}$ is an empirical parameter dependent on the experimental setup for measuring the transmission loss. Mulholland et al. [37] suggested the value of $\theta_{m}$ varying between $70^{\circ}$ and $85^{\circ}$, and Bolton et al. [14] chose the value of $72^{\circ}$ by matching their theoretical predictions to experimental results for an unlined single panel. This value of $\theta_{m}=72^{\circ}$ is used in the present study to calculate the transmission loss for the triple-panel structures. The integral of Eq. (33) is evaluated numerically using Simpson's rule in steps of $2^{\circ}$, and finally the random incidence transmission loss in a diffuse field is calculated through

$$
\mathrm{TL}=10 \log (1 / \bar{\tau})
$$

\section{Results and discussion}

\subsection{Parameters of the system}

To compare the performance of sound insulation between the doubleand triple-panel structures, the double-panel parameters used in Ref. [14] are taken in this paper to calculate transmission loss for the triple-panel structures, unless stated otherwise. In the experiments of Bolton et al. [14], aluminium sheets were used for the two elastic panels and a polyurethane foam slab as the porous material layer. The parameters of the aluminium panels and the polyurethane porous material are given in Table 2. Moreover, 
Table 2: Parameters used for the system [14].

\begin{tabular}{lll}
\hline Symbol & Description & Value \\
\hline Panel properties & \\
$\rho_{p}$ & material density & $2700 \mathrm{~kg} \mathrm{~m}^{-3}$ \\
$E_{p}$ & Young's modulus & $7 \times 10^{10} \mathrm{~Pa}$ \\
$\nu_{p}$ & Poisson's ratio & 0.33 \\
Porous & material properties & \\
$\rho_{1}$ & bulk density of solid phase & $30 \mathrm{~kg} \mathrm{~m}^{-3}$ \\
$E_{m}$ & bulk Young's modulus & $8 \times 10^{5} \mathrm{~Pa}$ \\
$\nu$ & bulk Poisson's ratio & 0.4 \\
$\eta$ & loss factor & 0.265 \\
$\sigma$ & flow resistivity & $25 \times 10^{3} \mathrm{MKS}$ Rayls m ${ }^{-1}$ \\
$\epsilon^{\prime}$ & geometrical structure factor & 7.8 \\
$h$ & porosity & 0.9 \\
\hline
\end{tabular}

the parameters relevant to the ambient air properties of standard atmosphere are: density $\rho_{0}=1.225 \mathrm{~kg} \mathrm{~m}^{-3}$, ratio of specific heats $\gamma=1.4$, Prandtle number $\operatorname{Pr}=0.71$, and speed of sound $c=340.3 \mathrm{~m} \mathrm{~s}^{-1}$. The two air gaps will take the ambient air parameters initially unless in the parametric study of Section 3.5.

The dimensions of the triple-panel structures will be different from those of the double-panel structures tested by Bolton et al. [14]. For these double-panel structures, the thickness of the first panel on the incident side is $h_{p 1}=1.27 \mathrm{~mm}$, and the second panel on the transmitted side is 
$h_{p 2}=0.76 \mathrm{~mm}$ in thickness; the thickness of the polyurethane foam is $L=27 \mathrm{~mm}$; the air gap of the BU configuration is $\delta=14 \mathrm{~mm}$ in depth, and the depths of the air gaps for the UU configuration are $2 \mathrm{~mm}$ and $6 \mathrm{~mm}$ on the incident and transmitted sides, respectively (see Fig. 2). For the triple-panel structures shown in Fig. 3, the thicknesses of the three panels are denoted by $h_{p 1}-h_{p 3}$, the two porous layers have thickness of $L_{1}, L_{2}$, and the two air gaps are $\delta_{1}, \delta_{2}$ in depth, respectively. To ensure a fair comparison of sound insulation performance, the total mass per unit area and total thickness of a triple-panel structure must be kept the same as those of its double-panel counterpart. This is achieved by satisfying equal total thicknesses of panel, foam and air gap respectively, for the two structures, i.e.

$$
\begin{gathered}
{ }^{2} h_{p 1}+{ }^{2} h_{p 2}={ }^{3} h_{p 1}+{ }^{3} h_{p 2}+{ }^{3} h_{p 3}, \\
L=L_{1}+L_{2}, \quad \delta=\delta_{1}+\delta_{2},
\end{gathered}
$$

where the left superscripts 2,3 denote a double- or a triple-panel structure. Following the conditions (35), the dimensions of a triple-panel structure is simply taken as: panel thickness, $h_{p 1}=h_{p 3}=0.76 \mathrm{~mm}, h_{p 2}=0.51 \mathrm{~mm}$; foam thickness, $L_{1}=L_{2}=L / 2=13.5 \mathrm{~mm}$; air gap depth, $\delta_{1}=\delta_{2}=\delta / 2=7 \mathrm{~mm}$. However for Category (ii) with only a single air gap, the depth remains the same as that of BU, i.e. $\delta=14 \mathrm{~mm}$.

\subsection{Model validation}

Two test cases are considered in order to validate the theoretical model and the numerical code employed in this study. Firstly, the results of a triple-panel structure without porous lining in the case of normal sound incidence are compared with the model predictions of Xin and Lu [26], as 


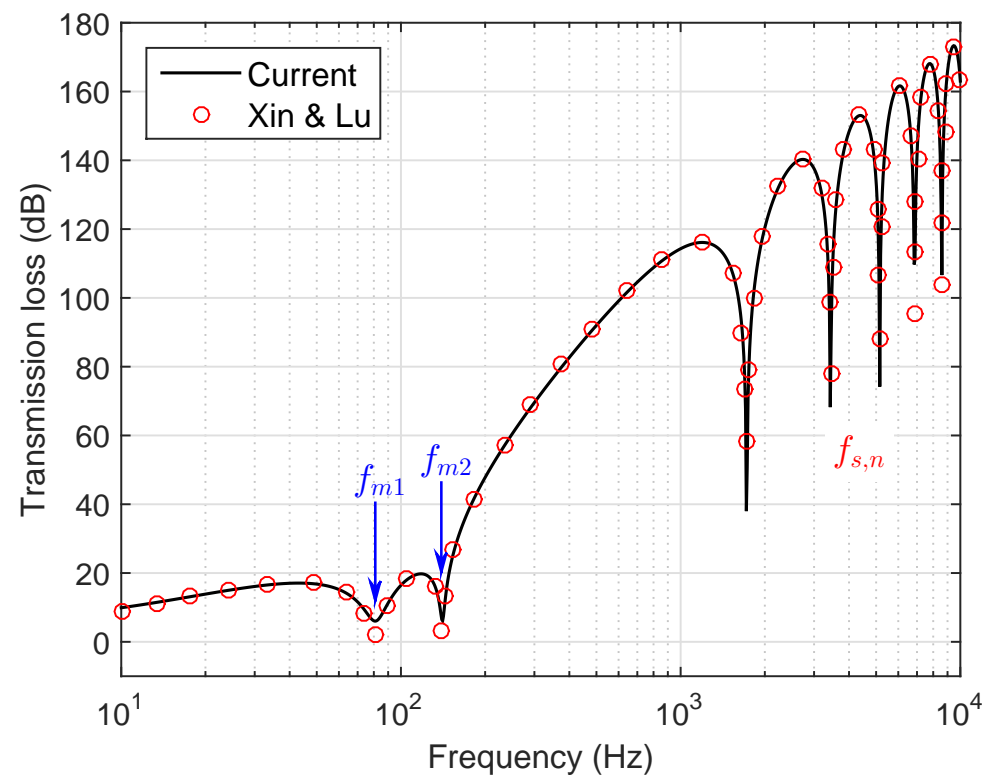

Figure 4: Comparison of transmission loss for a triple-panel structure without porous lining between current predictions and a previous model by Xin and $\mathrm{Lu}$ [26]. Panel and air gap dimensions are $\delta_{1}=\delta_{2}=0.1 \mathrm{~m}$ and $h_{p 1}=h_{p 2}=h_{p 3}=0.002 \mathrm{~m}$.

shown in Fig. 4. Two kinds of resonances were observed in their study, i.e., the 'mass-spring' resonance and the standing-wave resonance. The resonance frequencies of the equivalent 'mass-air-mass-air-mass' system were derived as [26]:

$$
f_{m 1,2}=\frac{\sqrt{2}}{4 \pi} \sqrt{\frac{\lambda_{1}+\lambda_{2} \mp \sqrt{\left(\lambda_{1}-\lambda_{2}\right)^{2}+4 \kappa_{1} \kappa_{2} m_{s 1}^{2} m_{s 3}^{2}}}{m_{s 1} m_{s 2} m_{s 3}}},
$$

where $\lambda_{1}=\kappa_{1} m_{s 3}\left(m_{s 1}+m_{s 2}\right)$ and $\lambda_{2}=\kappa_{2} m_{s 1}\left(m_{s 2}+m_{s 3}\right)$ are the equivalent stiffness of the two air gaps, respectively; $\kappa_{1,2}=\rho_{0} c^{2} / \delta_{1,2}$, and $m_{s i}(i=1,2,3)$ are the mass per unit area of the three panels, respectively. The standing-wave resonance occurs when the air gap depth matches integer numbers of the half wavelength of the incident sound, and 
the $n$-th standing-wave resonance frequency can be expressed as [38]:

$$
f_{s, n}=\frac{n c}{2 \delta}, \quad(n=1,2,3, \ldots)
$$

where in this test case the depth is $\delta=0.1 \mathrm{~m}$ for both air gaps.

As illustrated in Fig. 4, the present predictions agree excellently with those by Xin and $\mathrm{Lu}$ [26] particularly the resonance frequencies, which validates the theoretical model of the present study. The mass-spring resonance frequencies calculated from the formula in Eq. (36) are $82 \mathrm{~Hz}$ and $142 \mathrm{~Hz}$ for $f_{m 1}$ and $f_{m 2}$, respectively, and they are reproduced by both the present theory and the previous model in Ref. [26]. The standing-wave resonances occur at higher frequencies, i.e. $f_{s, n}=1715,3430,5145,6860 \mathrm{~Hz}$ from Eq. (37) for $n=1,2,3,4$ respectively, which are also captured accurately by the model predictions.

Secondly, the predictions of Bolton et al. [14] for double-panel structures lined with poroelastic materials were reproduced for the three configurations $\mathrm{BB}, \mathrm{BU}$ and UU. Figure 5 shows the predictions of the current study and Bolton et al. [14] as well as the experimental results in Ref. [14] for comparison. The excellent agreement between the three sets of results, in particular the prediction data, for all three configurations provides validation for the numerical code which will be applied to calculate the transmission loss for the triple-panel structures. The fundamental resonance frequency (i.e. mass-spring resonance frequency) of the classic problem of a double-panel structure without porous materials can be expressed as $[12,21]$ :

$$
f_{m}=\frac{1}{2 \pi} \sqrt{\frac{\rho_{0} c^{2}}{\delta} \frac{m_{s 1}+m_{s 2}}{m_{s 1} m_{s 2}}}
$$



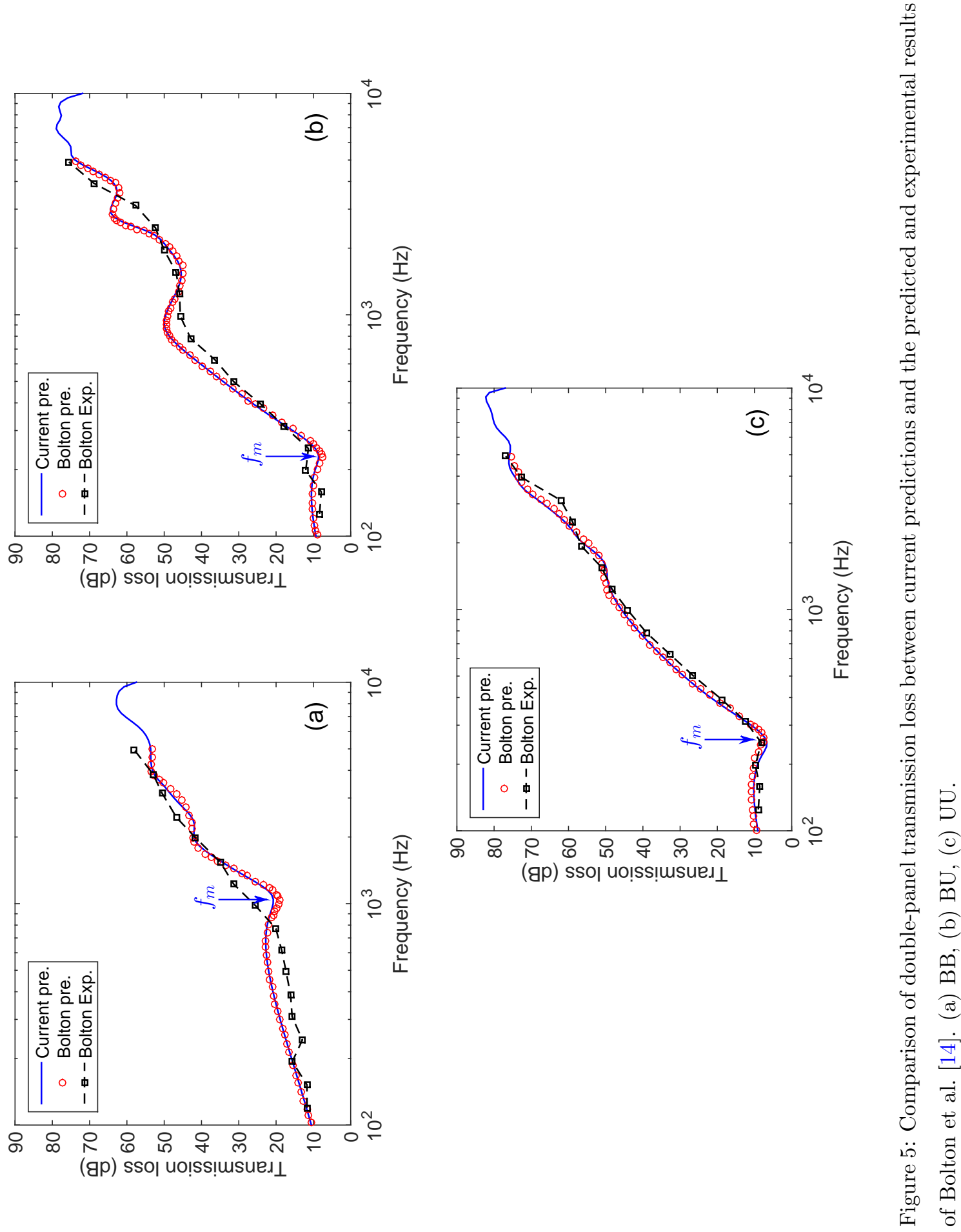
under normal incidence of sound. The values of $f_{m}$ for the BB, BU and UU configurations are calculated from Eq. (38) as $322 \mathrm{~Hz}, 262 \mathrm{~Hz}$ and $283 \mathrm{~Hz}$, respectively. As shown in Fig. 5, despite the effects of porous lining and random incidence, the fundamental resonance frequencies of the $\mathrm{BU}$ and $\mathrm{UU}$ cases are consistent with the predicted values from Eq. (38), whereas the BB resonance frequency increases to about $1000 \mathrm{~Hz}$ due to the stiffening effect of the directly attached porous lining on both sides [14]. These resonance dips, however, are well damped due to the sound attenuation by the porous foam and the averaging effect in a diffuse sound field.

\subsection{Comparison between double- and triple-panel structures}

A focus of this paper is to examine how a triple-panel structure performs in sound insulation compared with its double-panel counterpart. As aforementioned in Section 1, a triple-panel structure without porous linings offers a marked improvement only above a high cut-on frequency, i.e. the triple leaf effect. Two special cases, called the AA and AAAA configurations, in which only air gaps exist between the panels (i.e. $\delta=2 \delta_{1}=2 \delta_{2}=41 \mathrm{~mm}$ ) are hence considered to demonstrate this effect. The TL spectra of the two configurations are shown in Fig. 6 for comparison. The AA configuration retains the double-panel fundamental resonance frequency from Eq. (38) (i.e. $f_{m}=262 \mathrm{~Hz}$ ). The mass-spring resonance frequencies of the AAAA configuration are calculated from Eq. (36) as $f_{m 1}=292 \mathrm{~Hz}$ and $f_{m 2}=584 \mathrm{~Hz}$, respectively, and they are reproduced in the TL spectrum by the prediction model. However, the resonance dips are significantly damped due to random incidence effect in which the dip of $f_{m 2}$ nearly disappears.

As shown in Fig. 6, the transmission losses of AA and AAAA are almost 


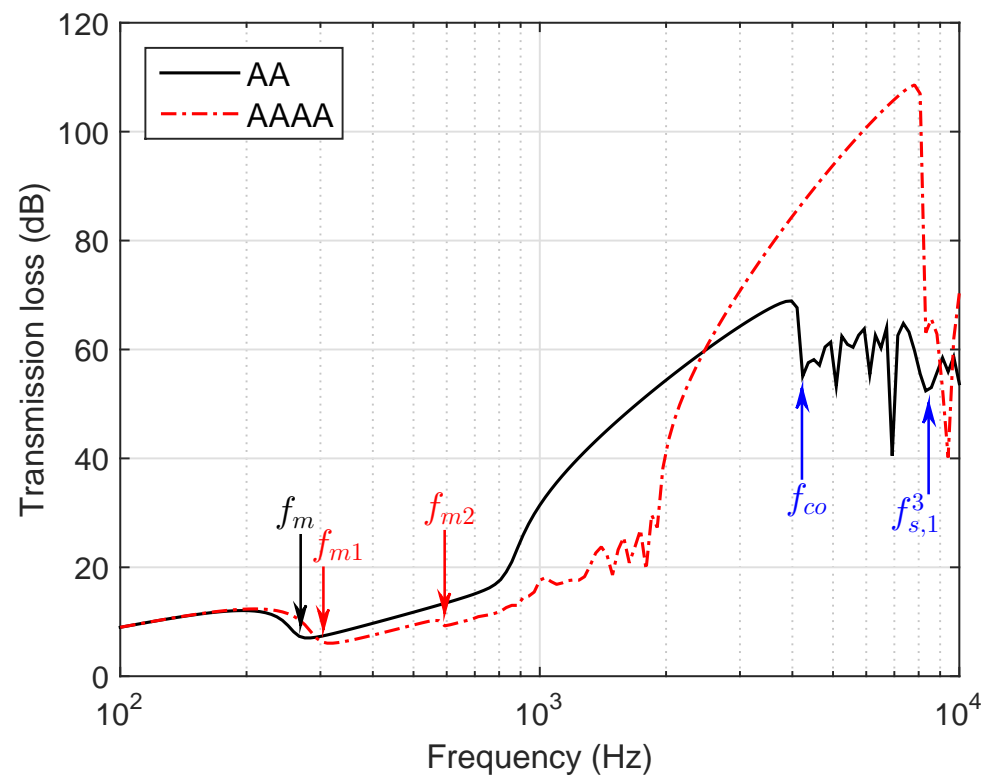

Figure 6: Transmission loss of a double- (AA) and a triple-panel (AAAA) structures without porous linings.

the same below $f_{m}$, consistent with the observation by Sharp [27, 29], and they follow approximately a mass-law behaviour based on the same total mass per unit area. However, because of the increased fundamental resonance frequency for AAAA, the double-panel configuration AA outperforms its triple-panel counterpart AAAA in most of the low-mid frequency range. From about $2000 \mathrm{~Hz}$ the transmission loss of AAAA starts to climb at a dramatic rate and it finally exceeds that of AA around $2500 \mathrm{~Hz}$. In this case, the cut-on frequency is equal to the first standing-wave resonance frequency of AA, i.e. $f_{c o}=f_{s, 1}^{2}=c / 2 \delta=4146 \mathrm{~Hz}$ from Eq. (37), and it is confirmed from Fig. 6 that above $f_{c o}$ the transmission loss of AAAA is noticeably higher than that of AA. In addition, the first standing-wave resonance frequency of 
AAAA (second resonance frequency for AA), i.e. $f_{s, 1}^{3}=f_{s, 2}^{2}=8293 \mathrm{~Hz}$ from Eq. (37), is also well captured by the model in Fig. 6.

The results of predicted transmission loss for the six typical triple-panel configurations are shown in Fig. 7. It is obvious that the sound insulation performance of the triple-panel configurations depends critically on the foam-panel coupling method (B or U), or more specifically on the number of air gaps in the structure. Therefore the three categories of triple-panel configurations, as defined in Section 2.3, exhibit distinct TL behaviours throughout the entire frequency range. The configurations of Category (ii) (BBBU and BUBB) show only one mass-spring resonance frequency because the ' $\mathrm{BB}$ ' bonding in one of the partitions reduces the structure to an equivalent 'mass-air-mass' system. This resonance frequency occurs around $300 \mathrm{~Hz}$, not much different from the theoretical value of $f_{m}=262 \mathrm{~Hz}$ for a double-panel structure. The Category (iii) configurations (BUBU, BUUB and UBBU) retain the two mass-spring resonance frequencies with well-damped resonance dips, typical of a triple-panel structure lined with poroelastic materials, which are close to the theoretical predictions $f_{m 1}=$ $292 \mathrm{~Hz}$ and $f_{m 2}=584 \mathrm{~Hz}$. Moreover, the reduced thickness of the foam layer and air gap for these triple-panel configurations results in very large standing-wave resonance frequencies (the lowest one above the frequency bound of $10000 \mathrm{~Hz}$ ), and hence these resonances will not be discussed hereafter.

With regard to the level of transmission loss, the BBBB case shows the highest transmission loss at low frequencies below $500 \mathrm{~Hz}$, but its high-frequency performance of sound insulation is evidently inferior to other 


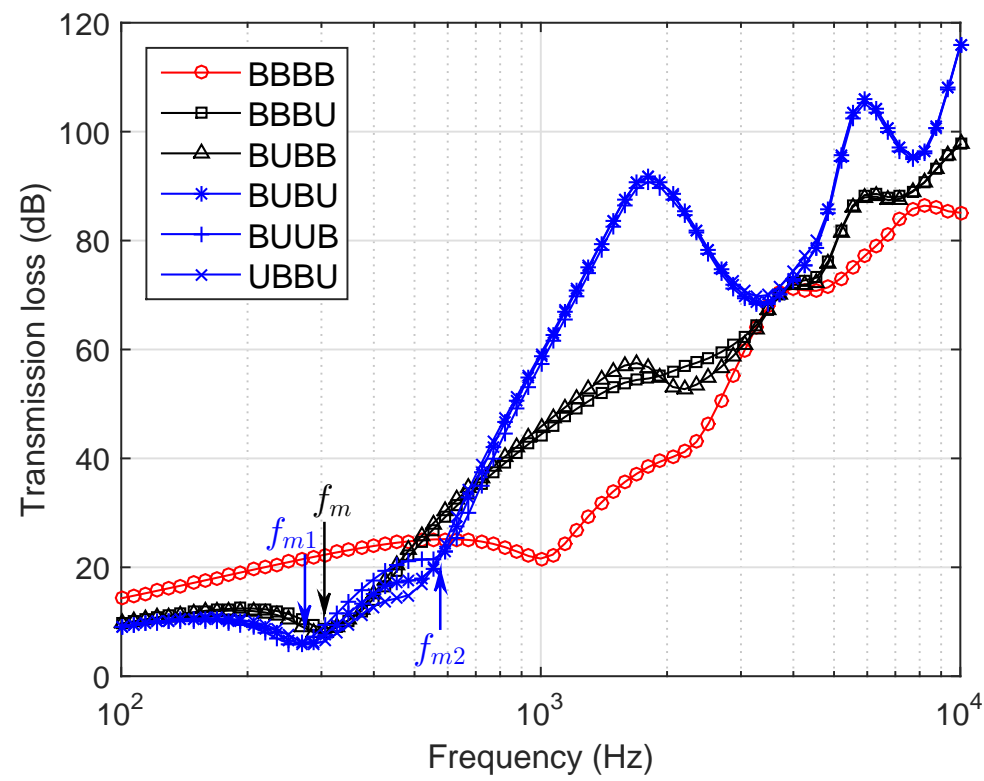

Figure 7: Transmission loss of six typical triple-panel structures.

configurations. This is because the direct foam-panel bonding at each interface makes the structure behaves as an entire mass system which has the highest overall stiffness and hence the best performance in the low-frequency range. For the same reason, the BBBB configuration retains the increased fundamental resonance frequency of the BB configuration around $1000 \mathrm{~Hz}$ as shown in Fig. 5(c). The BBBB configuration is favourable to the transmission of frame waves but not airborne waves since the frame wave in the foam layer is the dominant wave in such a configuration [14].

On the other hand, Category (iii) of configurations BUBU, BUUB and UBBU shows very close transmission loss throughout the entire frequency range except in the low-frequency band $\left[f_{m 1}, f_{m 2}\right]$ where BUUB shows a slight improvement of about $3 \mathrm{~dB}$ and $6 \mathrm{~dB}$ over the other two cases. The similar 
coupling methods of these configurations (i.e. two air gaps in the structure) account for the slight difference in their sound insulation performance. The relative higher transmission loss of BUUB within $\left[f_{m 1}, f_{m 2}\right]$ is owing to the enhanced stiffness of the two facing panels and hence the entire structure. In contrast to the BBBB configuration, Category (iii) shows poor low-frequency performance: below $f_{m 2}$ its transmission loss is lower than that of BBBB by up to $16 \mathrm{~dB}$. The two air gaps separate the structure physically into three detached elements, as shown in the bottom row of Fig. 3, which reduces the overall stiffness of the system and hence the low-frequency TL.

However, the striking feature for this category of configurations is the remarkably superior performance of sound insulation in the mid-high frequency range. As can be seen in Fig. 7, above $700 \mathrm{~Hz}$ the transmission loss of Category (iii) is distinctly higher than those of Categories (i) and (ii) by up to $53 \mathrm{~dB}$ and $35 \mathrm{~dB}$, respectively, and it displays a sharp $45 \mathrm{~dB} /$ octave slope except for the troughs around $3500 \mathrm{~Hz}$ and $7700 \mathrm{~Hz}$. The existence of two narrow air gaps in Category (iii) configurations increases the number of layers in the structure and as a result more wave reflections occur at the layer interfaces, which ultimately enhances the attenuation of high-frequency sound within the poroelastic materials. Moreover, the configurations BBBU and BUBB in Category (ii) also show similar TL spectra which lie between those of the other two categories in almost the entire frequency range. Considering the sound insulation performance over the entire frequency range, therefore, Category (iii) in particular BUUB may be the overall best structure since this category provides the highest transmission loss in the mid-high frequency range yet with an acceptable sacrifice of performance in 


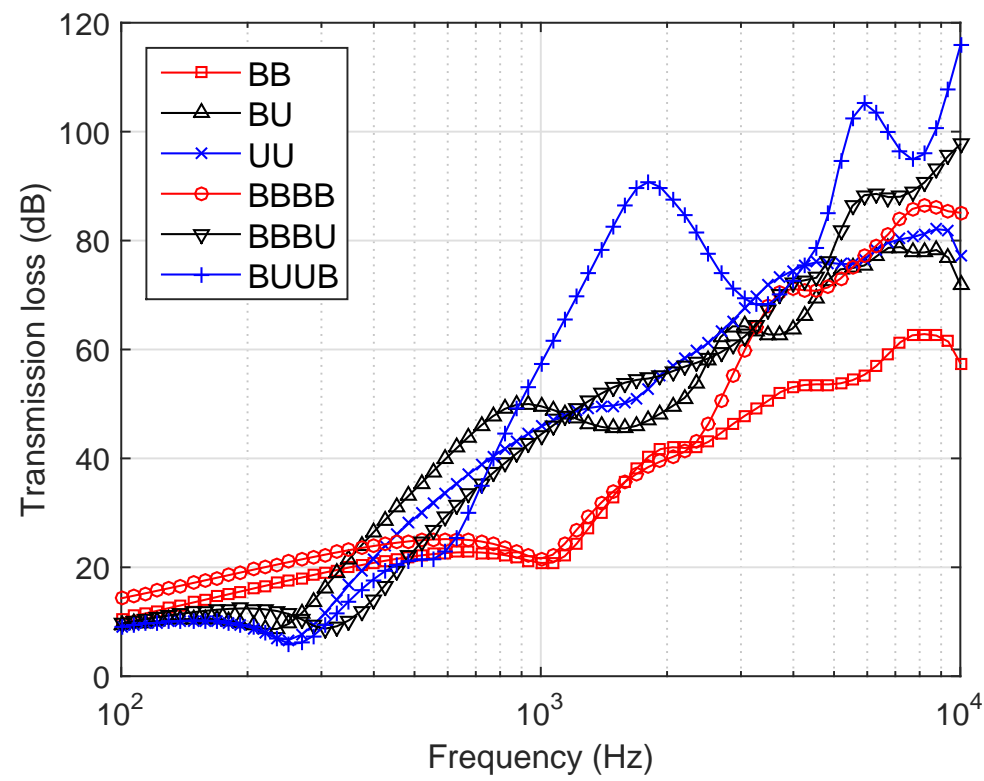

Figure 8: Comparison of transmission loss between typical double- and triple-panel structures.

the low-frequency range.

The next step is to compare the sound insulation properties of the doubleand triple-panel structures lined with poroelastic materials. Figure 8 shows the transmission loss of the three double-panel configurations (BB, BU and UU) and three typical triple-panel configurations from Categories (i)-(iii), i.e. $\mathrm{BBBB}, \mathrm{BBBU}$ and BUUB. The comparison is drawn between each triple-panel category and its double-panel counterpart, respectively. At low frequencies the BBBB configuration is still the most efficient structure which outperforms its double-panel counterpart BB in TL by about $3.5 \mathrm{~dB}$ below $600 \mathrm{~Hz}$. Although both configurations, BBBB and BB, have the same mass per unit area, the extra middle panel of BBBB improves the 
system stiffness and hence the low-frequency performance. The BBBB configuration also shows higher transmission loss than BB by up to $28 \mathrm{~dB}$ at frequencies above $2300 \mathrm{~Hz}$ which is almost halved compared with the cut-on frequency for the case of AAAA vs. AA. This is because the middle panel of BBBB induces extra reflected waves (and hence more absorption) within the porous material and the thin foam layers are favourable to high-frequency reflection. Note that the fundamental resonance frequency of BBBB is supposed to be increased by the stiffening effect but remains unchanged at $1000 \mathrm{~Hz}$ because the dramatic climb of transmission loss at high frequencies apparently elevates the TL values in the neighbouring mid-frequency range. Consider next the configurations BU and BBBU. The TL trends of the two configurations appear very similar. However, the fundamental resonance frequency of BBBU shifts upward to $310 \mathrm{~Hz}$ because the structure is reinforced somewhat by the extra middle panel, which results in a TL 7-12 dB lower than that of $\mathrm{BU}$ from the resonance frequency to $1000 \mathrm{~Hz}$. The two configurations then take the lead in TL alternatively, and at high frequencies above $3300 \mathrm{~Hz}$ the configuration BBBU outperforms BU significantly due to the enhanced multiple wave reflections and the reduced size of the foam layers by the middle panel, as have been explained for the case of BBBB and BB. Finally, the UU configuration shows similar TL as BU [14], and the BUUB configuration still yields the best high-frequency performance; above $900 \mathrm{~Hz}$ (22\% of the cut-on frequency for AAAA and AA) the transmission loss of BUUB is higher than those of UU and BU by up to $44 \mathrm{~dB}$.

To sum up, in general the sound transmission loss of a triple-panel configuration is higher than that of its double-panel counterpart in the 
mid-high frequency range. The porous lining is capable of lowering the cut-on frequency where the triple-panel configurations noticeably outperform their double-panel counterparts and hence extends the applicability of a triple-panel structure to lower frequencies. The high-frequency TL of the triple-panel configurations are hence remarkably increased by the combination of the triple-panel structure and the porous lining. In addition, a triple-panel configuration can also outperform its double-panel counterpart at low frequencies, for example BBBB vs. $\mathrm{BB}$ and $\mathrm{BBBU}$ vs. BU. The different methods of attaching the porous lining to the panel (i.e. bonded or unbonded) yield a variety of triple-panel configurations. In practice, an appropriate configuration with the optimal overall performance can be selected according to the target frequency range of sound insulation, which is an obvious advantage of a triple-panel structure over a double-panel structure.

\subsection{Effect of foam and air gap thicknesses}

The effects of material parameters on the transmission loss of the double-panel structures have been presented in Ref. [14]. The parametric effects on a triple-panel structure will be similar to the double-panel results provided the porous materials are uniform in both layers. A simple example is the effect of foam thickness on the transmission loss of the doubleand triple-panel configurations: BB, BU, BBBB and BUBU. For BB and BBBB, the total foam thickness $L$ varies from $15 \mathrm{~mm}$ to $39 \mathrm{~mm}$ in step of $4 \mathrm{~mm}$ and equal thickness is assumed for the two foam layers of BBBB, i.e. $L_{1}=L_{2}=L / 2$. For BU and BUBU, the same variation of foam thickness is applied and the total thickness of the structure remains unchanged as 
that in previous analysis $(41 \mathrm{~mm})$, i.e. the air gap depth is decreasing correspondingly as the foam thickness increases; equal air gap depth is assumed for BUBU, i.e. $\delta_{1}=\delta_{2}=\delta / 2$.

Figures $9(\mathrm{a})-9(\mathrm{~d})$ show the transmission loss of the four configurations with varying foam thickness (and air gap depth). As expected, the TL variation of a triple-panel structure appears similar to that of the double-panel counterpart. For the configurations $\mathrm{BB}$ and $\mathrm{BBBB}$, the increasing foam thickness shifts the TL curves to lower frequencies because the fundamental resonance frequency is reduced due to the enlarged depth between the two facing panels. Hence as the foam thickness increases, the soundproof performance of $\mathrm{BB}$ and $\mathrm{BBBB}$ is improved at high frequencies but with a slight TL reduction in the low-frequency range of 400-1000 Hz. For the configurations BU and BUUB, however, the resonance frequency is nearly unaffected by the varying foam thickness since the total thickness of the structure is fixed as $41 \mathrm{~mm}$. The increasing foam thickness (and decreasing air gap depth) also shifts the TL curves downward in frequency along with the reduced magnitude of the local peaks. This effect is more evident for the BUUB configuration. As shown in Figs. 9(b) and 9(d), the first peak of BU falls from $1600 \mathrm{~Hz}$ to $650 \mathrm{~Hz}$ by $18 \mathrm{~dB}$ when the foam thickness varies from $15 \mathrm{~mm}$ to $39 \mathrm{~mm}$, whereas for BUUB the drop occurs from $3300 \mathrm{~Hz}$ to $1250 \mathrm{~Hz}$ by $30 \mathrm{~dB}$. It is therefore more effective to tune the acoustic performance of a triple-panel structure by simply varying the ratio of foam thickness to air gap depth. Note that even when the total air gap is extremely thin, i.e. $\delta=2 \mathrm{~mm}$, the configurations BU and BUUB still greatly outperform $\mathrm{BB}$ and $\mathrm{BBBB}$ at frequencies from about $400 \mathrm{~Hz}$, 

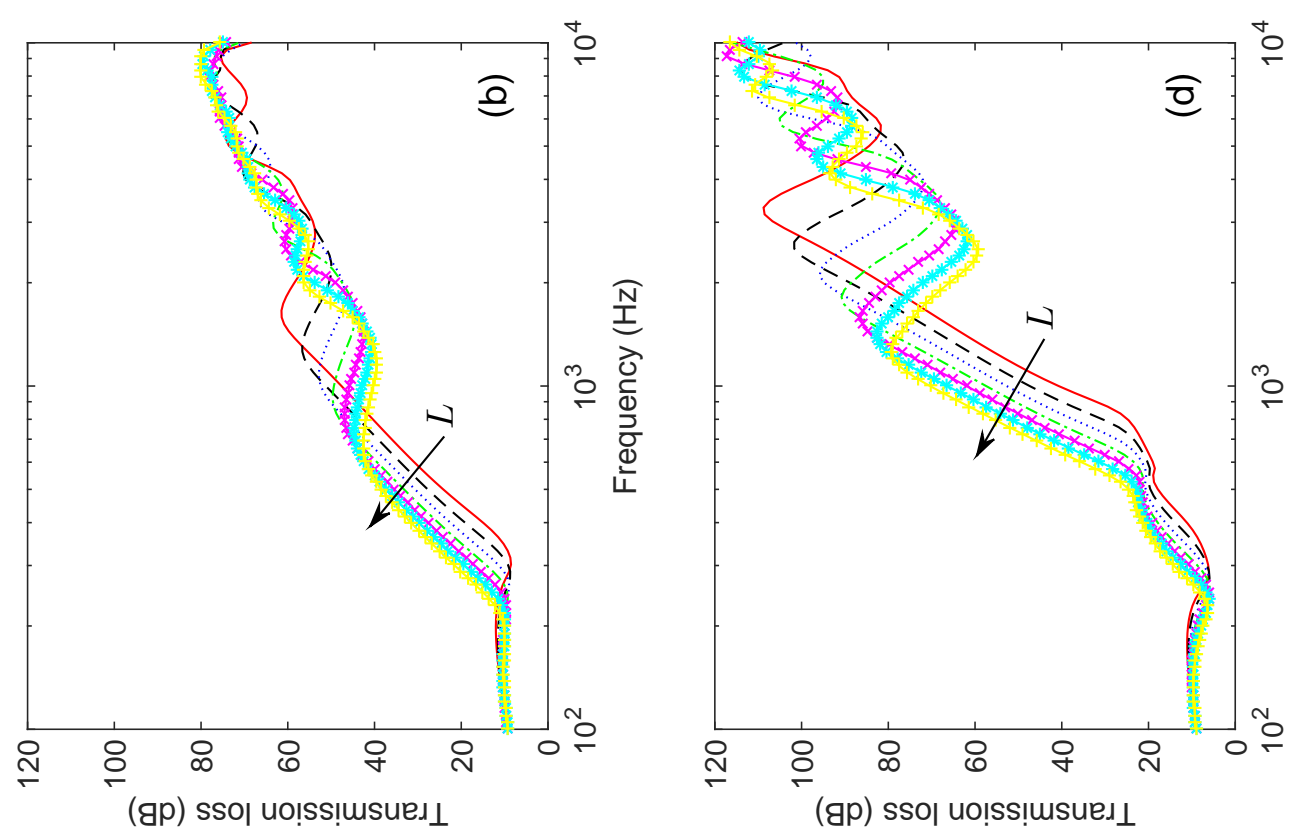

0.
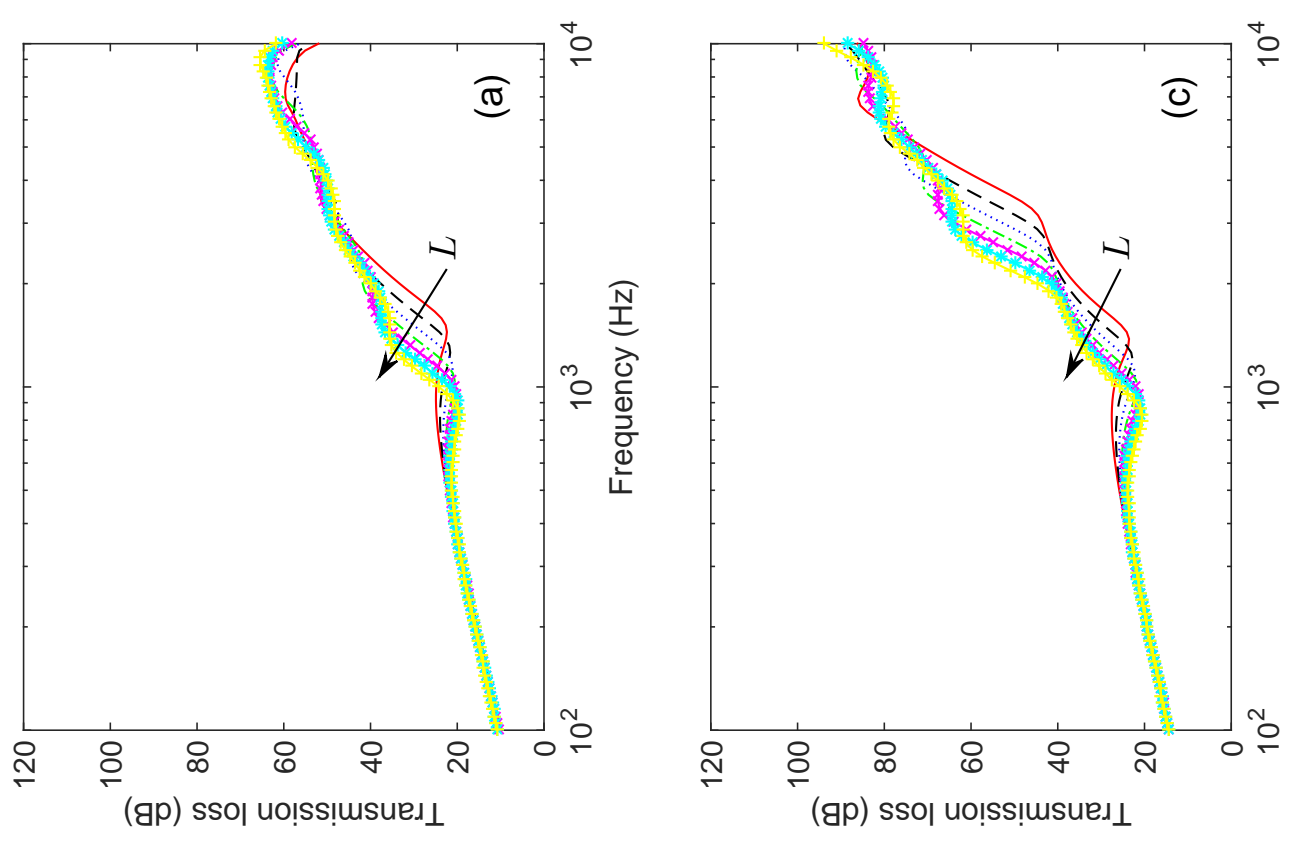

0ิ 
which emphasises the importance of the air gap to the sound insulation of a multiple-panel structure. Additionally, if there is no constraint on the total thickness of the structure, it is anticipated that a thicker structure will further improve the low-frequency soundproof performance.

An advantage of a triple-panel structure is that the foam and air properties in the two partitions can vary independently. For instance, assuming that $L_{1}=2 L_{2}$ and $\delta_{1}=2 \delta_{2}$ while keeping other relations (e.g. variations of $L$ and $\delta$ ) as in the previous case, the effect of foam thickness (and air gap depth) on the transmission loss of the BBBB and BUUB configurations is shown in Fig. 10. The results of BBBB are nearly unchanged compared to those in Fig. 9(c), which indicates that the position of the middle panel has minor effect on the soundproof performance of a triple-panel structure. The transmission loss of BUUB in Fig. 10(b) shows little variation to that in Fig. 9(d), neither, at low frequencies below $1000 \mathrm{~Hz}$ since the total foam thickness and total air gap depth are varying identically between the two figures. However, the non-uniform thicknesses of foam and air gap in the two partitions appear to attenuate significantly the local peaks (and troughs) observed in Fig. 9(d), resulting in much less oscillatory TL behaviours in the high-frequency range. Furthermore, alternative methods are also available to achieve various control effects according to the target of sound insulation; for example the foam thickness can increase in one partition whereas decrease in the other partition. Therefore, the extra partition of a triple-panel structure with air gaps provides more design space for tuning the sound insulation performance at high frequencies. 


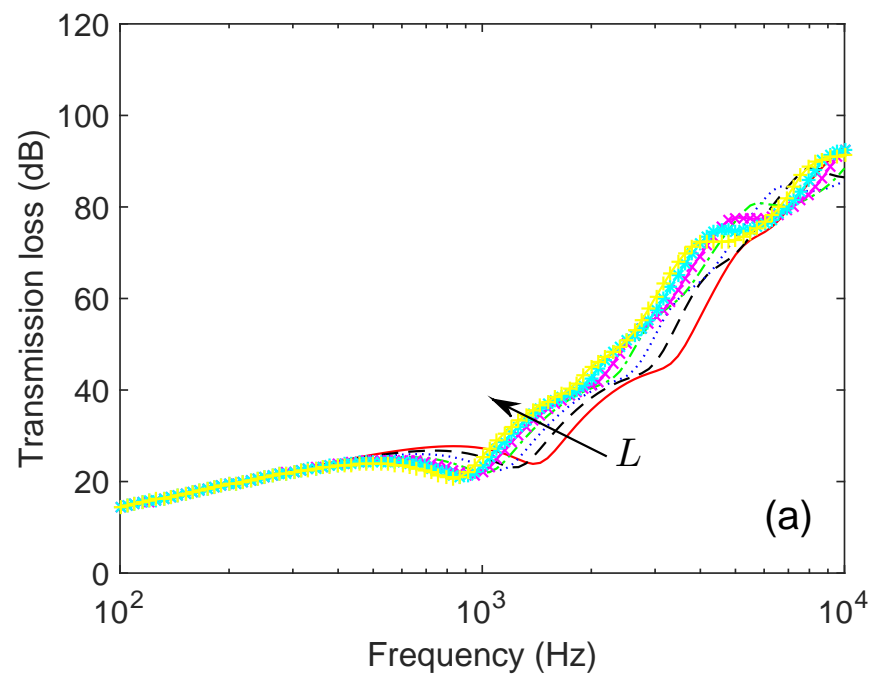

(a)

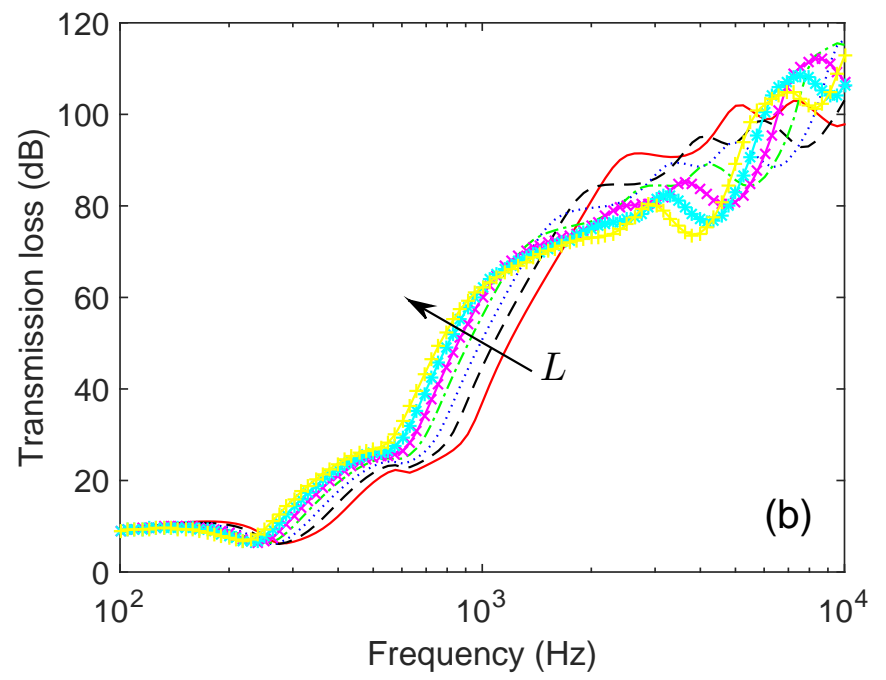

Figure 10: Transmission loss of the (a) BBBB and (b) BUUB configurations with different varying thicknesses of the two foam layers. 
Table 3: The properties of air at various altitudes [39] to be applied in the air gaps.

\begin{tabular}{cccc} 
Altitude $(\mathrm{m})$ & Density $\left(\mathrm{kg} \mathrm{m}^{-3}\right)$ & Sound speed $\left(\mathrm{m} \mathrm{s}^{-1}\right)$ & Prandtle number \\
\hline 3000 & 0.909 & 328.6 & 0.715 \\
6000 & 0.660 & 316.5 & 0.720 \\
10000 & 0.414 & 299.5 & 0.729 \\
\hline
\end{tabular}

\subsection{Effect of air gap pressure}

Apart from the thicknesses of the foam layers and air gaps, the porous material parameters can be varied to adjust the transmission loss and the results for the double-panel structures can be found in Ref. [14]. Compared with the material parameters, altering the air properties and hence the acoustic impedance in the air gaps (and within the interior of adjacent porous layers) is relatively easy to implement in practice for the purpose of active control. The mismatch of acoustic impedance between the air gap and ambient air on the incident and transmitted sides has been observed to increase the transmission loss $[20,40,41]$. In this section, the effect of pressure in the air gaps is studied by considering air properties at different altitudes as shown in Table 3, while the the ambient air remains the standard atmosphere at sea level. The triple-panel dimensions discussed in Section 3.1 are employed in the numerical calculation. Note that in this case all incident waves will transmit through the air gaps because the decreased acoustic impedance of the air gaps reduces the incidence angle according to the Snell-Descartes law in Eq. (7).

Figures 11(a)-11(d) show the effect of air gap pressure on the transmission 

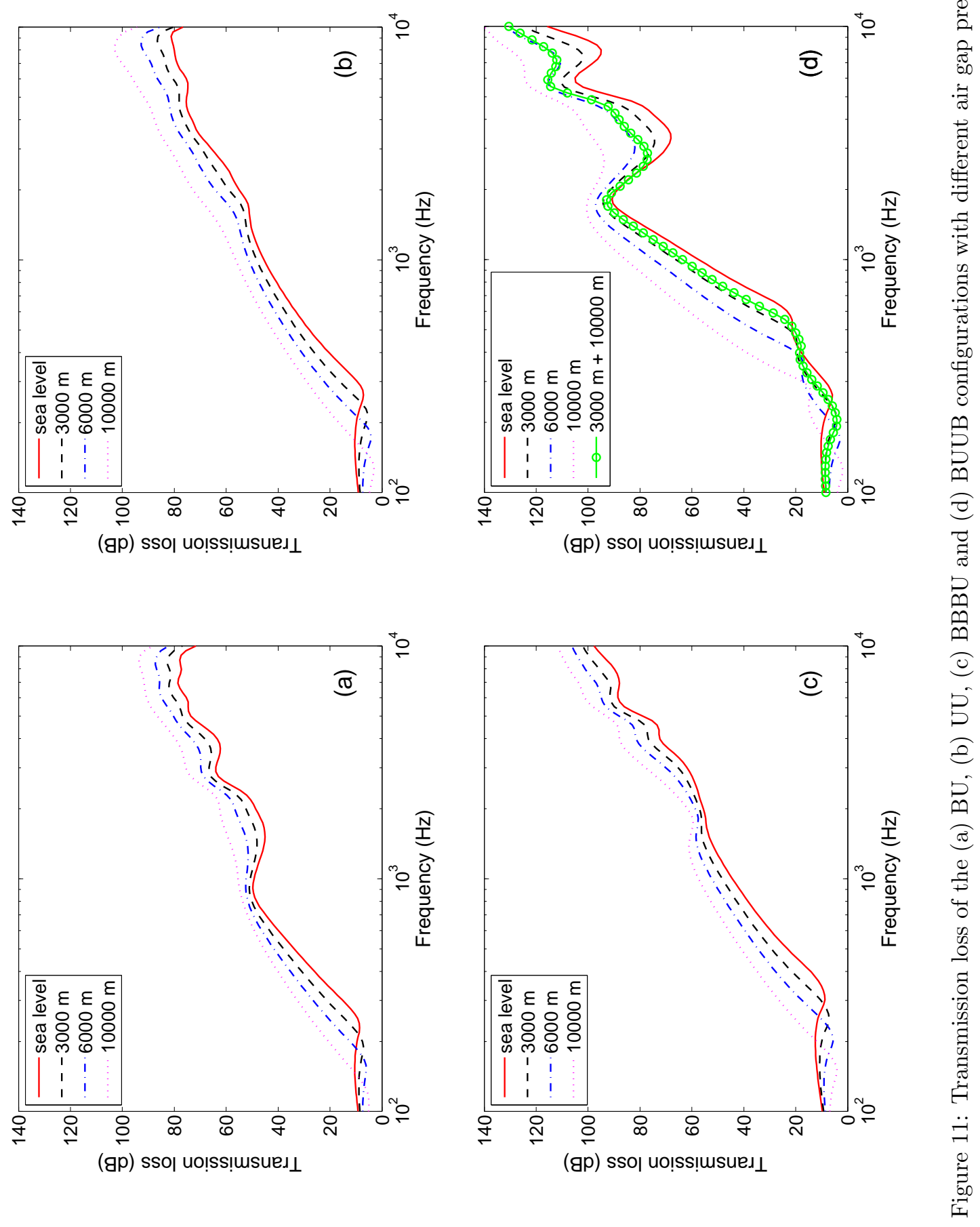
loss of the double- and triple-panel configurations, i.e. BU, UU, BBBU and BUUB. The air properties at higher altitudes reduce the acoustic impedance and hence the fundamental resonance frequency. As a result of the mismatch of acoustic impedance, the TL curve for air properties at sea level is shifted downward to lower frequencies and the TL levels are elevated significantly from the resonance frequencies. As can be observed in Fig. 11, the effect of air gap pressure on the TL variations appears to be very similar among the configurations $\mathrm{BU}, \mathrm{UU}$ and BBBU. It is obvious that the BUUB configuration benefits more from the impedance mismatch than the other three configurations, which is a merit of the triple-panel structure with two air gaps. As the pressure in the air gap decreases from sea level to an altitude of $10000 \mathrm{~m}$, the TL level increases by about $5-17 \mathrm{~dB}$ for BU, $11-22 \mathrm{~dB}$ for UU, 5-20 dB for BBBU and 9-33 dB for BUUB, respectively. Moreover, the TL troughs observed for the air properties at sea level, characteristic of the BUUB configuration, are disappearing gradually with lower air gap pressures, which results in an even higher increase of transmission loss at these frequencies.

The two air gaps in the BUUB configuration also allow air with different properties existing in them, and similarly it is supposed to provide an additional option for the control of sound insulation. However, Fig. 11(d) shows that using different air properties at altitudes of $3000 \mathrm{~m}$ and $10000 \mathrm{~m}$ in the two air gaps does not gain extra benefits. Below $3000 \mathrm{~Hz}$ the TL of this case is very close to that of air properties at $3000 \mathrm{~m}$ and then it approximates the TL of air properties of $6000 \mathrm{~m}$ at very high frequencies. As aforementioned, the improvement of sound insulation performance depends 
on the difference of acoustic impedance between the air gaps and ambient air. Compared with the case of uniform air properties of $10000 \mathrm{~m}$ in both air gaps, applying properties of air at $3000 \mathrm{~m}$ and $10000 \mathrm{~m}$ in the two air gaps separately diminishes the overall degree of impedance mismatch and hence the transmission loss falls off. In addition, the application of different air gap pressures further increases the system complexity and is therefore not recommended as an effective control method to implement in practice.

\section{Conclusions}

In this work, a theoretical model [14] to predict the transmission loss of a double-panel structure lined with poroelastic materials has been extended to the case of triple-panel structures. Biot's theory [15] has been applied in the model to describe wave propagations in poroelastic materials. The extended model has been validated by reproducing the predicted and measured transmission losses of the double-panel configurations in Ref. [14]. Based on the methods of mounting the foam layers to the panels, a triple-panel structure with an extra partition provides more configurations than its double-panel counterpart. Six typical triple-panel configurations were considered in this study and they can be divided into three categories according to the number of 'unbonded' coupling in the structure. It has been confirmed that the foam-panel coupling method, or more specifically the number of air gaps in a triple-panel structure, has a profound influence on the sound insulation performance. The configurations in Category (iii), i.e. BUBU, BUUB and UBBU, yield the optimal overall performance with the remarkably high transmission loss in the mid-high frequency range, whereas 
the BBBB configuration shows better performance at low frequencies; the transmission loss of Category (ii) with a single air gap falls between the results of the other two categories.

Compared with a double-panel structure having the same total mass per unit area and total thickness, a triple-panel structure generally exhibits better soundproof performance in the high-frequency range and the porous lining extends this advantage to significantly lower frequencies, which eliminates the drawback of a triple-panel wall with a noticeable improvement in TL only above a high cut-on frequency, i.e. the triple leaf effect. A triple-panel structure can also outperform its double-panel counterpart in the low-frequency range if the appropriate configurations are selected. The variety of configurations provided by a triple-panel structure hence provides more design space to meet a target frequency range of sound insulation in practice, and there is always a triple-panel configuration superior to the double-panel counterpart throughout the entire frequency range.

The properties of the foam layers and air gaps can be varied to tune the sound insulation performance of a triple-panel structure. Varying the foam and air gap thicknesses is observed to be more effective for a triple-panel structure than for a double-panel structure. It has also been shown that air properties of higher altitudes in the air gaps produce higher transmission loss and a triple-panel structure is more sensitive to this effect than its double-panel counterpart. The triple-panel structure also allows the foam and air properties to vary independently in the two partitions, which provides more control options for its soundproof performance. However, applying different air properties in the two air gaps is not recommended as it reduces 
the average impedance difference between the air gaps and ambient air without additional benefit.

Although complexity is an inherent drawback, the triple-panel structure with poroelastic linings has great potential to replace the double-panel sandwich structure widely used in practice without the penalty in weight or volume because this structure has superior performance of sound insulation, particularly in the mid-high frequency range, and provides more space for control purpose. It can be anticipated that better performance at higher frequencies yet with a greater TL sacrifice in the low-frequency range can be achieved through a multiple-panel structure with even more layers when necessary. In this case, an optimisation study using a transfer matrix method needs to be carried out to maximise the transmission loss within the imposed constraints of weight and volume. The presence of external mean flow and air gap flow has a profound influence on sound transmission through a realistic multiple-panel structure and turns the system into a three-dimensional problem, which will be addressed in future work.

\section{Acknowledgements}

The author would like to acknowledge Prof. J. Stuart Bolton at Purdue University and Mr Jie Zhou at the University of Southampton for their help on the poroelastic material parameters during the validation of the numerical code. The efforts of Mr Olivier Marchand and Mr Craig Ferns made to the initial code are acknowledged. The author thanks the anonymous reviewers for their valuable comments to this paper. 


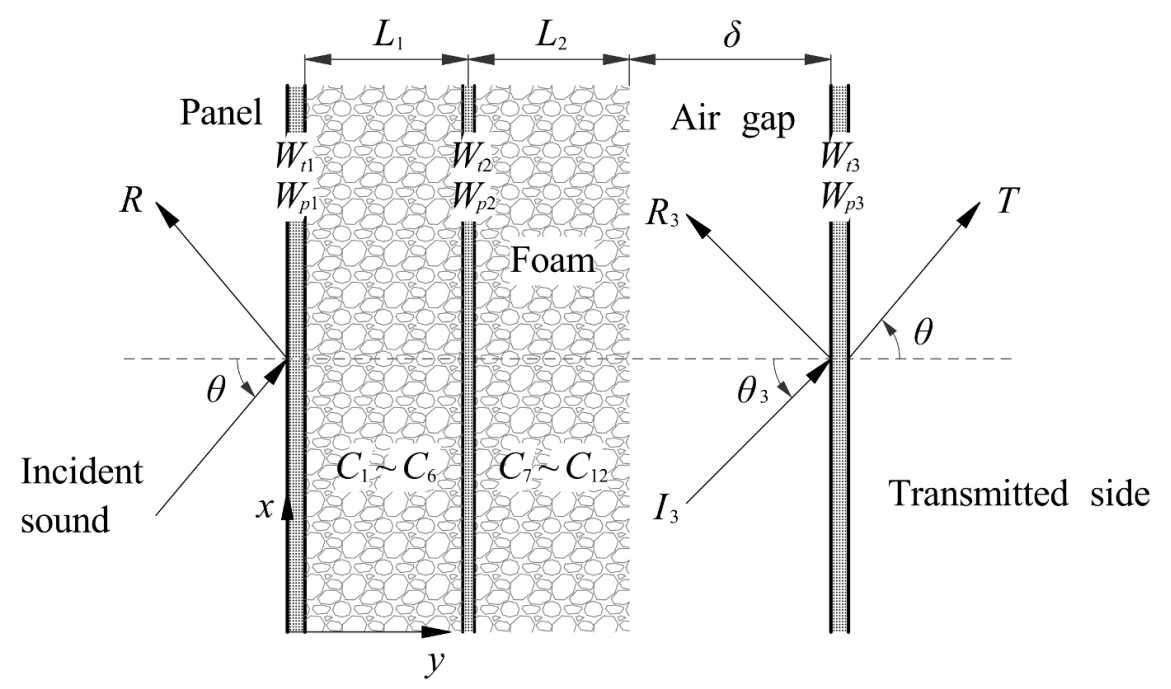

Figure A.1: Schematic of sound transmission through the BBBU configuration.

\section{Appendix A. An example of the transfer matrix equation}

The BBBU configuration is chosen as an example to demonstrate in detail the derivation of the matrix equation and the transfer coefficients using appropriate boundary conditions. This configuration requires all four types of boundary conditions discussed in Section 2.3, including BC (IV) which is characteristic of a triple- or multiple-panel structure. Figure A.1 illustrates schematically sound transmission through the BBBU configuration.

The different types of boundary conditions to be satisfied for the BBBU configuration are listed as follows.

$\mathrm{BC}(\mathrm{II})$ at $y=0$ :

$$
\begin{aligned}
& \text { (1) } v_{y 1}=-\frac{\partial \phi_{1}}{\partial y}=\mathrm{j} \omega W_{t 1}, \quad \text { (2) } u_{y 1}=W_{t 1}, \quad \text { (3) } U_{y 1}=W_{t 1}, \\
& \text { (4) } u_{x 1}=W_{p 1}-\mathrm{j} k_{x} \frac{h_{p 1}}{2} W_{t 1}, \quad \text { (5) } \tau_{y x 1}=\left(D_{p 1} k_{x}^{2}-\omega^{2} m_{s 1}\right) W_{p 1} \\
& \text { (6) } p_{1}+\sigma_{y 1}+s_{1}-\mathrm{j} k_{x} \frac{h_{p 1}}{2} \tau_{x y 1}=\left(D_{1} k_{x}^{4}-\omega^{2} m_{s 1}\right) W_{t 1} .
\end{aligned}
$$




$$
\begin{aligned}
& \text { (7) } u_{y 1}=W_{t 2}, \quad(8) U_{y 1}=W_{t 2}, \quad(9) u_{y 2}=W_{t 2}, \quad(10) U_{y 2}=W_{t 2}, \\
& \text { (11) } u_{x 1}=W_{p 2}+\mathrm{j} k_{x} \frac{h_{p 2}}{2} W_{t 2}, \quad(12) u_{x 2}=W_{p 2}-\mathrm{j} k_{x} \frac{h_{p 2}}{2} W_{t 2}, \\
& \text { (13) } \tau_{y x 2}-\tau_{y x 1}=\left(D_{p 2} k_{x}^{2}-\omega^{2} m_{s 2}\right) W_{p 2}, \\
& \text { (14) }\left(\sigma_{y 1}+s_{1}\right)-\left(\sigma_{y 2}+s_{2}\right)-\mathrm{j} k_{x} \frac{h_{p 2}}{2}\left(\tau_{x y 1}+\tau_{x y 2}\right)=\left(D_{2} k_{x}^{4}-\omega^{2} m_{s 2}\right) W_{t 2} .
\end{aligned}
$$

$\mathrm{BC}(\mathrm{I})$ at $y=L_{1}+L_{2}$ :

$$
\begin{aligned}
& (15)-h p_{3}=-h\left(\mathrm{j} \omega \rho_{3} \phi_{3}\right)=s_{2}, \\
& (16)-(1-h) p_{3}=-(1-h)\left(\mathrm{j} \omega \rho_{3} \phi_{3}\right)=\sigma_{y 2}, \\
& (17) v_{y 3}=-\frac{\partial \phi_{2}}{\partial y}=\mathrm{j} \omega(1-h) u_{y 2}+\mathrm{j} \omega h U_{y 2}, \quad \text { (18) } \tau_{x y 2}=0 .
\end{aligned}
$$

$\mathrm{BC}(\mathrm{III})$ at $y=L_{1}+L_{2}+\delta$ :

$$
\begin{aligned}
& \text { (19) } v_{y 3}=-\frac{\partial \phi_{3}}{\partial y}=\mathrm{j} \omega W_{t 3}, \quad(20) v_{y 4}=-\frac{\partial \phi_{t}}{\partial y}=\mathrm{j} \omega W_{t 3}, \\
& \text { (21) } p_{3}-p_{4}=\mathrm{j} \omega\left(\rho_{3} \phi_{3}-\rho_{0} \phi_{t}\right)=\left(D_{3} k_{x}^{4}-\omega^{2} m_{s 3}\right) W_{t 3} .
\end{aligned}
$$

In the above $\mathrm{BC}$ equations, the velocity potentials $\phi_{1}, \phi_{3}, \phi_{t}$ have been expressed in Eqs. (3)-(5) that includes the reflection and transmission coefficients $R$ and $T$; the transverse and in-plane displacements of the three panels, $W_{t 1,2,3}$ and $W_{p 1,2,3}$, can be found in Eqs. (8) and (9); the solutions of $u_{x 1,2}, u_{y 1,2}, U_{x 1,2}, U_{y 1,2}, \sigma_{y 1,2}, s_{1,2}, \tau_{x y 1,2}$ containing the constants $C_{1}-C_{12}$ for the two foam layers were given by Eqs. (22) and (26). Substituting these equations into the boundary conditions (A.1)-(A.4), a transfer matrix equation can be obtained as

$$
\mathbf{A}_{21} \mathbf{C}_{21}=\mathbf{B}_{21}
$$


where the $i$ th row of equation corresponds to the $i$ th boundary condition. The vector of unknown amplitudes is:

$$
\mathbf{C}_{21}^{T}=\left[C_{1}, C_{2}, \ldots, C_{12}, I_{3}, R_{3}, W_{t 1}, W_{t 2}, W_{t 3}, W_{p 1}, W_{p 2}, R, T\right]
$$

The non-zero elements of the forcing vector $\mathbf{B}$ are $\mathbf{B}(1)=k_{y}$ and $\mathbf{B}(6)=$ $-\mathrm{j} \omega \rho_{0}$. The non-zero elements of the transfer coefficient matrix $\mathbf{A}$ are:

$$
\begin{aligned}
& \mathbf{A}(1,15)=\omega, \quad \mathbf{A}(1,20)=k_{y} ; \quad \mathbf{A}(2,1)=k_{\mathrm{I} y} / k_{\mathrm{I}}^{2}, \quad \mathbf{A}(2,2)=-k_{\mathrm{I} y} / k_{\mathrm{I}}^{2}, \\
& \mathbf{A}(2,3)=k_{\mathrm{II} y} / k_{\mathrm{II}}^{2}, \quad \mathbf{A}(2,4)=-k_{\mathrm{II} y} / k_{\mathrm{II}}^{2}, \quad \mathbf{A}(2,5)=k_{x} / k_{r}^{2}, \quad \mathbf{A}(2,6)=k_{x} / k_{r}^{2}, \\
& \mathbf{A}(2,15)=\mathrm{j} ; \quad \mathbf{A}(3,1)=\left(k_{\mathrm{I} y} / k_{\mathrm{I}}^{2}\right) b_{1}, \quad \mathbf{A}(3,2)=-\left(k_{\mathrm{I} y} / k_{\mathrm{I}}^{2}\right) b_{1}, \\
& \mathbf{A}(3,3)=\left(k_{\mathrm{II} y} / k_{\mathrm{II}}^{2}\right) b_{2}, \quad \mathbf{A}(3,4)=-\left(k_{\mathrm{II} y} / k_{\mathrm{II}}^{2}\right) b_{2}, \quad \mathbf{A}(3,5)=\left(k_{x} / k_{r}^{2}\right) g, \\
& \mathbf{A}(3,6)=\left(k_{x} / k_{r}^{2}\right) g, \quad \mathbf{A}(3,15)=\mathrm{j} ; \quad \mathbf{A}(4,1)=k_{x} / k_{\mathrm{I}}^{2}, \quad \mathbf{A}(4,2)=k_{x} / k_{\mathrm{I}}^{2}, \\
& \mathbf{A}(4,3)=k_{x} / k_{\mathrm{II}}^{2}, \quad \mathbf{A}(4,4)=k_{x} / k_{\mathrm{II}}^{2}, \quad \mathbf{A}(4,5)=-k_{t y} / k_{r}^{2}, \quad \mathbf{A}(4,6)=k_{t y} / k_{r}^{2}, \\
& \mathbf{A}(4,15)=-k_{x} h_{p 1} / 2, \quad \mathbf{A}(4,18)=\mathrm{j} ; \quad \mathbf{A}(5,1)=2 k_{\mathrm{I} y} k_{x} / k_{\mathrm{I}}^{2}, \quad \mathbf{A}(5,2)=-2 k_{\mathrm{I} y} k_{x} / k_{\mathrm{I}}^{2}, \\
& \mathbf{A}(5,3)=2 k_{\mathrm{II} y} k_{x} / k_{\mathrm{II}}^{2}, \quad \mathbf{A}(5,4)=-2 k_{\mathrm{II} y} k_{x} / k_{\mathrm{II}}^{2}, \quad \mathbf{A}(5,5)=\left(k_{x}^{2}-k_{r y}^{2}\right) / k_{r}^{2}, \\
& \mathbf{A}(5,6)=\left(k_{x}^{2}-k_{r y}^{2}\right) / k_{r}^{2}, \quad \mathbf{A}(5,18)=\left(\omega^{2} m_{s 1}-D_{p 1} k_{x}^{2}\right) / N ; \\
& \mathbf{A}(6,1)=2 N k_{\mathrm{I} y}^{2} / k_{\mathrm{I}}^{2}+A+Q+b_{1} E_{2}-\mathrm{j} N h_{p 1} k_{x}^{2} k_{\mathrm{I} y} / k_{\mathrm{I}}^{2}, \\
& \mathbf{A}(6,2)=2 N k_{\mathrm{I} y}^{2} / k_{\mathrm{I}}^{2}+A+Q+b_{1} E_{2}+\mathrm{j} N h_{p 1} k_{x}^{2} k_{\mathrm{I} y} / k_{\mathrm{I}}^{2}, \\
& \mathbf{A}(6,3)=2 N k_{\mathrm{II} y}^{2} / k_{\mathrm{II}}^{2}+A+Q+b_{1} E_{2}-\mathrm{j} N h_{p 1} k_{x}^{2} k_{\mathrm{II} y} / k_{\mathrm{II}}^{2}, \\
& \mathbf{A}(6,4)=2 N k_{\mathrm{II} y}^{2} / k_{\mathrm{II}}^{2}+A+Q+b_{1} E_{2}+\mathrm{j} N h_{p 1} k_{x}^{2} k_{\mathrm{II} y} / k_{\mathrm{II}}^{2}, \\
& \mathbf{A}(6,5)=2 N k_{x} k_{r y} / k_{r}^{2}-\mathrm{j} N h_{p 1} k_{x}\left(k_{x}^{2}-k_{r y}^{2}\right) /\left(2 k_{r}^{2}\right), \\
& \mathbf{A}(6,6)=-2 N k_{x} k_{r y} / k_{r}^{2}-\mathrm{j} N h_{p 1} k_{x}\left(k_{x}^{2}-k_{r y}^{2}\right) /\left(2 k_{r}^{2}\right), \\
& \mathbf{A}(6,15)=\omega^{2} m_{s 1}-D_{1} k_{x}^{4}, \quad \mathbf{A}(6,20)=\mathrm{j} \omega \rho_{0} ; \\
& \mathbf{A}(7,1)=\left(k_{\mathrm{I} y} / k_{\mathrm{I}}^{2}\right) \mathrm{e}^{-\mathrm{j} k_{\mathrm{I} y} L_{1}}, \quad \mathbf{A}(7,2)=-\left(k_{\mathrm{I} y} / k_{\mathrm{I}}^{2}\right) \mathrm{e}^{\mathrm{j} k_{\mathrm{I} y} L_{1}},
\end{aligned}
$$




$$
\begin{aligned}
& \mathbf{A}(7,3)=\left(k_{\mathrm{II} y} / k_{\mathrm{II}}^{2}\right) \mathrm{e}^{-\mathrm{j} k_{\mathrm{II} y} L_{1}}, \quad \mathbf{A}(7,4)=-\left(k_{\mathrm{II} y} / k_{\mathrm{II}}^{2}\right) \mathrm{e}^{\mathrm{j} k_{\mathrm{II} y} L_{1}}, \\
& \mathbf{A}(7,5)=\left(k_{x} / k_{r}^{2}\right) \mathrm{e}^{-\mathrm{j} k_{r y} L_{1}}, \quad \mathbf{A}(7,6)=\left(k_{x} / k_{r}^{2}\right) \mathrm{e}^{\mathrm{j} k_{r y} L_{1}}, \quad \mathbf{A}(7,16)=\mathbf{j} ; \\
& \mathbf{A}(8,1)=\left(k_{\mathrm{I} y} / k_{\mathrm{I}}^{2}\right) b_{1} \mathrm{e}^{-\mathrm{j} k_{\mathrm{I} y} L_{1}}, \quad \mathbf{A}(8,2)=-\left(k_{\mathrm{I} y} / k_{\mathrm{I}}^{2}\right) b_{1} \mathrm{e}^{\mathrm{j} k_{\mathrm{I} y} L_{1}}, \\
& \mathbf{A}(8,3)=\left(k_{\mathrm{II} y} / k_{\mathrm{II}}^{2}\right) b_{2} \mathrm{e}^{-\mathrm{j} k_{\mathrm{II} y} L_{1}}, \quad \mathbf{A}(8,4)=-\left(k_{\mathrm{II} y} / k_{\mathrm{II}}^{2}\right) b_{2} \mathrm{e}^{\mathrm{j} k_{\mathrm{II} y} L_{1}}, \\
& \mathbf{A}(8,5)=\left(k_{x} / k_{r}^{2}\right) g \mathrm{e}^{-\mathrm{j} k_{r y} L_{1}}, \quad \mathbf{A}(8,6)=\left(k_{x} / k_{r}^{2}\right) g \mathrm{e}^{\mathrm{j} k_{r y} L_{1}}, \quad \mathbf{A}(8,16)=\mathrm{j} ; \\
& \mathbf{A}(9,1)=\left(k_{x} / k_{\mathrm{I}}^{2}\right) \mathrm{e}^{-\mathrm{j} k_{\mathrm{I} y} L_{1}}, \quad \mathbf{A}(9,2)=\left(k_{x} / k_{\mathrm{I}}^{2}\right) \mathrm{e}^{\mathrm{j} k_{\mathrm{I} y} L_{1}}, \\
& \mathbf{A}(9,3)=\left(k_{x} / k_{\mathrm{II}}^{2}\right) \mathrm{e}^{-\mathrm{j} k_{\mathrm{II} y} L_{1}}, \quad \mathbf{A}(9,4)=\left(k_{x} / k_{\mathrm{II}}^{2}\right) \mathrm{e}^{\mathrm{j} k_{\mathrm{II} y} L_{1}}, \\
& \mathbf{A}(9,5)=-\left(k_{r y} / k_{r}^{2}\right) \mathrm{e}^{-\mathrm{j} k_{r y} L_{1}}, \quad \mathbf{A}(9,6)=\left(k_{r y} / k_{r}^{2}\right) \mathrm{e}^{\mathrm{j} k_{r y} L_{1}}, \quad \mathbf{A}(9,16)=k_{x} h_{p 2} / 2, \\
& \mathbf{A}(9,19)=\mathrm{j} ; \quad \mathbf{A}(10,7)=\left(k_{\mathrm{I} y} / k_{\mathrm{I}}^{2}\right) \mathrm{e}^{-\mathrm{j} k_{\mathrm{I} y} L_{1}}, \quad \mathbf{A}(10,8)=-\left(k_{\mathrm{I} y} / k_{\mathrm{I}}^{2}\right) \mathrm{e}^{\mathrm{j} k_{\mathrm{I} y} L_{1}}, \\
& \mathbf{A}(10,9)=\left(k_{\mathrm{II} y} / k_{\mathrm{II}}^{2}\right) \mathrm{e}^{-\mathrm{j} k_{\mathrm{II} y} L_{1}}, \quad \mathbf{A}(10,10)=-\left(k_{\mathrm{II} y} / k_{\mathrm{II}}^{2}\right) \mathrm{e}^{\mathrm{j} k_{\mathrm{II} y} L_{1}}, \\
& \mathbf{A}(10,11)=\left(k_{x} / k_{r}^{2}\right) \mathrm{e}^{-\mathrm{j} k_{r y} L_{1}}, \quad \mathbf{A}(10,12)=\left(k_{x} / k_{r}^{2}\right) \mathrm{e}^{\mathrm{j} k_{r y} L_{1}}, \quad \mathbf{A}(10,16)=\mathrm{j} ; \\
& \mathbf{A}(11,7)=\left(k_{\mathrm{I} y} / k_{\mathrm{I}}^{2}\right) \mathrm{e}^{-\mathrm{j} k_{\mathrm{I} y} L_{1}}, \quad \mathbf{A}(11,8)=-\left(k_{\mathrm{I} y} / k_{\mathrm{I}}^{2}\right) \mathrm{e}^{\mathrm{j} k_{\mathrm{I} y} L_{1}}, \\
& \mathbf{A}(11,9)=\left(k_{\mathrm{II} y} / k_{\mathrm{II}}^{2}\right) \mathrm{e}^{-\mathrm{j} k_{\mathrm{II} y} L_{1}}, \quad \mathbf{A}(11,10)=-\left(k_{\mathrm{II} y} / k_{\mathrm{II}}^{2}\right) \mathrm{e}^{\mathrm{j} k_{\mathrm{II} y} L_{1}}, \\
& \mathbf{A}(11,11)=\left(k_{x} / k_{r}^{2}\right) \mathrm{e}^{-\mathrm{j} k_{r y} L_{1}}, \quad \mathbf{A}(11,12)=\left(k_{x} / k_{r}^{2}\right) \mathrm{e}^{\mathrm{j} k_{r y} L_{1}}, \quad \mathbf{A}(11,16)=\mathbf{j} ; \\
& \mathbf{A}(12,7)=\left(k_{x} / k_{\mathrm{I}}^{2}\right) \mathrm{e}^{-\mathrm{j} k_{\mathrm{I} y} L_{1}}, \quad \mathbf{A}(12,8)=\left(k_{x} / k_{\mathrm{I}}^{2}\right) \mathrm{e}^{\mathrm{j} k_{\mathrm{I} y} L_{1}}, \\
& \mathbf{A}(12,9)=\left(k_{x} / k_{\mathrm{II}}^{2}\right) \mathrm{e}^{-\mathrm{j} k_{\mathrm{II} y} L_{1}}, \quad \mathbf{A}(12,10)=\left(k_{x} / k_{\mathrm{II}}^{2}\right) \mathrm{e}^{\mathrm{j} k_{\mathrm{II} y} L_{1}} \\
& \mathbf{A}(12,11)=-\left(k_{r y} / k_{r}^{2}\right) \mathrm{e}^{-\mathrm{j} k_{r y} L_{1}}, \quad \mathbf{A}(12,12)=\left(k_{r y} / k_{r}^{2}\right) \mathrm{e}^{\mathrm{j} k_{r y} L_{1}}, \\
& \mathbf{A}(12,16)=-k_{x} h_{p 2} / 2, \quad \mathbf{A}(12,19)=\mathrm{j} ; \\
& \mathbf{A}(13,1)=-2 k_{\mathrm{I} y} k_{x} / k_{\mathrm{I}}^{2} \mathrm{e}^{-\mathrm{j} k_{\mathrm{I}} L_{1}}, \quad \mathbf{A}(13,2)=2 k_{\mathrm{Iy}} k_{x} / k_{\mathrm{I}}^{2} \mathrm{e}^{\mathrm{j} k_{\mathrm{Iy}} L_{1}}, \\
& \mathbf{A}(13,3)=-2 k_{\mathrm{II} y} k_{x} / k_{\mathrm{II}}^{2} \mathrm{e}^{-\mathrm{j} k_{\mathrm{II} y} L_{1}}, \quad \mathbf{A}(13,4)=2 k_{\mathrm{II} y} k_{x} / k_{\mathrm{II}}^{2} \mathrm{e}^{\mathrm{j} k_{\mathrm{II} y} L_{1}}, \\
& \mathbf{A}(13,5)=-\left(k_{x}^{2}-k_{r y}^{2}\right) / k_{r}^{2} \mathrm{e}^{-\mathrm{j} k_{r y} L_{1}}, \quad \mathbf{A}(13,6)=-\left(k_{x}^{2}-k_{r y}^{2}\right) / k_{r}^{2} \mathrm{e}^{\mathrm{j} k_{r y} L_{1}}, \\
& \mathbf{A}(13,7)=2 k_{\mathrm{I} y} k_{x} / k_{\mathrm{I}}^{2} \mathrm{e}^{-\mathrm{j} k_{\mathrm{I} y} L_{1}}, \quad \mathbf{A}(13,8)=-2 k_{\mathrm{I} y} k_{x} / k_{\mathrm{I}}^{2} \mathrm{e}^{\mathrm{j} k_{\mathrm{I} y} L_{1}},
\end{aligned}
$$


$\mathbf{A}(13,9)=2 k_{\mathrm{II} y} k_{x} / k_{\mathrm{II}}^{2} \mathrm{e}^{-\mathrm{j} k_{\mathrm{II} y} L_{1}}, \quad \mathbf{A}(13,10)=-2 k_{\mathrm{II} y} k_{x} / k_{\mathrm{II}}^{2} \mathrm{e}^{\mathrm{j} k_{\mathrm{II} y} L_{1}}$, $\mathbf{A}(13,11)=\left(k_{x}^{2}-k_{r y}^{2}\right) / k_{r}^{2} \mathrm{e}^{-\mathrm{j} k_{r y} L_{1}}, \quad \mathbf{A}(13,12)=\left(k_{x}^{2}-k_{r y}^{2}\right) / k_{r}^{2} \mathrm{e}^{\mathrm{j} k_{r y} L_{1}}$,

$$
\mathbf{A}(13,19)=\left(\omega^{2} m_{s 2}-D_{p 2} k_{x}^{2}\right) / N
$$

$\mathbf{A}(14,1)=\left(-2 N k_{\mathrm{I} y}^{2} / k_{\mathrm{I}}^{2}+A+Q+b_{1} E_{2}-\mathrm{j} N h_{p 2} k_{x}^{2} k_{\mathrm{I} y} / k_{\mathrm{I}}^{2}\right) \mathrm{e}^{-\mathrm{j} k_{\mathrm{I} y} L_{1}}$, $\mathbf{A}(14,2)=\left(-2 N k_{\mathrm{I} y}^{2} / k_{\mathrm{I}}^{2}+A+Q+b_{1} E_{2}+\mathrm{j} N h_{p 2} k_{x}^{2} k_{\mathrm{I} y} / k_{\mathrm{I}}^{2}\right) \mathrm{e}^{\mathrm{j} k_{\mathrm{I} y} L_{1}}$, $\mathbf{A}(14,3)=\left(-2 N k_{\mathrm{II} y}^{2} / k_{\mathrm{II}}^{2}+A+Q+b_{2} E_{2}-\mathrm{j} N h_{p 2} k_{x}^{2} k_{\mathrm{II} y} / k_{\mathrm{II}}^{2}\right) \mathrm{e}^{-\mathrm{j} k_{\mathrm{II} y} L_{1}}$, $\mathbf{A}(14,4)=\left(-2 N k_{\mathrm{II} y}^{2} / k_{\mathrm{II}}^{2}+A+Q+b_{2} E_{2}+\mathrm{j} N h_{p 2} k_{x}^{2} k_{\mathrm{II} y} / k_{\mathrm{II}}^{2}\right) \mathrm{e}^{\mathrm{j} k_{\mathrm{II} y} L_{1}}$, $\mathbf{A}(14,5)=\left[-2 N k_{x} k_{r y} / k_{r}^{2}-\mathrm{j} N h_{p 2} k_{x}\left(k_{x}^{2}-k_{r y}^{2}\right) /\left(2 k_{r}^{2}\right)\right] \mathrm{e}^{-\mathrm{j} k_{r y} L_{1}}$, $\mathbf{A}(14,6)=\left[2 N k_{x} k_{r y} / k_{r}^{2}-\mathrm{j} N h_{p 2} k_{x}\left(k_{x}^{2}-k_{r y}^{2}\right) /\left(2 k_{r}^{2}\right)\right] \mathrm{e}^{\mathrm{j} k_{r y} L_{1}}$, $\mathbf{A}(14,7)=\left(2 N k_{\mathrm{I} y}^{2} / k_{\mathrm{I}}^{2}+A+Q+b_{1} E_{2}-\mathrm{j} N h_{p 2} k_{x}^{2} k_{\mathrm{I} y} / k_{\mathrm{I}}^{2}\right) \mathrm{e}^{-\mathrm{j} k_{\mathrm{I} y} L_{1}}$, $\mathbf{A}(14,8)=\left(2 N k_{\mathrm{I} y}^{2} / k_{\mathrm{I}}^{2}+A+Q+b_{1} E_{2}+\mathrm{j} N h_{p 2} k_{x}^{2} k_{\mathrm{I} y} / k_{\mathrm{I}}^{2}\right) \mathrm{e}^{\mathrm{j} k_{\mathrm{I} y} L_{1}}$, $\mathbf{A}(14,9)=\left(2 N k_{\mathrm{II} y}^{2} / k_{\mathrm{II}}^{2}+A+Q+b_{2} E_{2}-\mathrm{j} N h_{p 2} k_{x}^{2} k_{\mathrm{II} y} / k_{\mathrm{II}}^{2}\right) \mathrm{e}^{-\mathrm{j} k_{\mathrm{II} y} L_{1}}$, $\mathbf{A}(14,10)=\left(2 N k_{\mathrm{II} y}^{2} / k_{\mathrm{II}}^{2}+A+Q+b_{2} E_{2}+\mathrm{j} N h_{p 2} k_{x}^{2} k_{\mathrm{II} y} / k_{\mathrm{II}}^{2}\right) \mathrm{e}^{\mathrm{j} k_{\mathrm{II} y} L_{1}}$, $\mathbf{A}(14,11)=\left[2 N k_{x} k_{r y} / k_{r}^{2}-\mathrm{j} N h_{p 2} k_{x}\left(k_{x}^{2}-k_{r y}^{2}\right) /\left(2 k_{r}^{2}\right)\right] \mathrm{e}^{-\mathrm{j} k_{r y} L_{1}}$, $\mathbf{A}(14,12)=\left[-2 N k_{x} k_{r y} / k_{r}^{2}-\mathrm{j} N h_{p 2} k_{x}\left(k_{x}^{2}-k_{r y}^{2}\right) /\left(2 k_{r}^{2}\right)\right] \mathrm{e}^{\mathrm{j} k_{r y} L_{1}}$,

$$
\mathbf{A}(14,16)=\omega^{2} m_{s 2}-D_{2} k_{x}^{4}
$$

$$
\mathbf{A}(15,7)=\left(Q+b_{1} R^{\prime}\right) \mathrm{e}^{-\mathrm{j} k_{\mathrm{I} y}\left(L_{1}+L_{2}\right)}, \quad \mathbf{A}(15,8)=\left(Q+b_{1} R^{\prime}\right) \mathrm{e}^{\mathrm{j} k_{\mathrm{I} y}\left(L_{1}+L_{2}\right)},
$$$$
\mathbf{A}(15,9)=\left(Q+b_{2} R^{\prime}\right) \mathrm{e}^{-\mathrm{j} k_{\mathrm{II} y}\left(L_{1}+L_{2}\right)}, \quad \mathbf{A}(15,10)=\left(Q+b_{2} R^{\prime}\right) \mathrm{e}^{\mathrm{j} k_{\mathrm{II} y}\left(L_{1}+L_{2}\right)},
$$

$$
\begin{gathered}
\mathbf{A}(15,13)=\mathrm{j} \omega \rho_{3} h \mathrm{e}^{-\mathrm{j} k_{3 y}\left(L_{1}+L_{2}\right)}, \quad \mathbf{A}(15,14)=\mathrm{j} \omega \rho_{3} h \mathrm{e}^{\mathrm{j} k_{3 y}\left(L_{1}+L_{2}\right)} \\
\mathbf{A}(16,7)=\left(2 N k_{\mathrm{I} y}^{2} / k_{\mathrm{I}}^{2}+A+b_{1} Q\right) \mathrm{e}^{-\mathrm{j} k_{\mathrm{I} y}\left(L_{1}+L_{2}\right)} \\
\mathbf{A}(16,8)=\left(2 N k_{\mathrm{I} y}^{2} / k_{\mathrm{I}}^{2}+A+b_{1} Q\right) \mathrm{e}^{\mathrm{j} k_{\mathrm{I} y}\left(L_{1}+L_{2}\right)} \\
\mathbf{A}(16,9)=\left(2 N k_{\mathrm{II} y}^{2} / k_{\mathrm{II}}^{2}+A+b_{2} Q\right) \mathrm{e}^{-\mathrm{j} k_{\mathrm{II} y}\left(L_{1}+L_{2}\right)}
\end{gathered}
$$




$$
\begin{aligned}
& \mathbf{A}(16,10)=\left(2 N k_{\mathrm{II} y}^{2} / k_{\mathrm{II}}^{2}+A+b_{2} Q\right) \mathrm{e}^{\mathrm{j} k_{\mathrm{II} y}\left(L_{1}+L_{2}\right)}, \\
& \mathbf{A}(16,11)=2 N\left(k_{x} k_{r y} / k_{r}^{2}\right) \mathrm{e}^{-\mathrm{j} k_{r y}\left(L_{1}+L_{2}\right)}, \quad \mathbf{A}(16,12)=-2 N\left(k_{x} k_{r y} / k_{r}^{2}\right) \mathrm{e}^{\mathrm{j} k_{r y}\left(L_{1}+L_{2}\right)}, \\
& \mathbf{A}(16,13)=\mathrm{j} \omega \rho_{3}(1-h) \mathrm{e}^{-\mathrm{j} k_{3 y}\left(L_{1}+L_{2}\right)}, \quad \mathbf{A}(16,14)=\mathrm{j} \omega \rho_{3}(1-h) \mathrm{e}^{\mathrm{j} k_{3 y}\left(L_{1}+L_{2}\right)} ; \\
& \mathbf{A}(17,7)=-\omega\left(1-h+h b_{1}\right)\left(k_{\mathrm{I} y} / k_{\mathrm{I}}^{2}\right) \mathrm{e}^{-\mathrm{j} k_{\mathrm{I}}\left(L_{1}+L_{2}\right)}, \\
& \mathbf{A}(17,8)=\omega\left(1-h+h b_{1}\right)\left(k_{\mathrm{I} y} / k_{\mathrm{I}}^{2}\right) \mathrm{e}^{\mathrm{j} k_{\mathrm{I} y}\left(L_{1}+L_{2}\right)}, \\
& \mathbf{A}(17,9)=-\omega\left(1-h+h b_{2}\right)\left(k_{\mathrm{II} y} / k_{\mathrm{II}}^{2}\right) \mathrm{e}^{-\mathrm{j} k_{\mathrm{II} y}\left(L_{1}+L_{2}\right)}, \\
& \mathbf{A}(17,10)=\omega\left(1-h+h b_{2}\right)\left(k_{\mathrm{II} y} / k_{\mathrm{II}}^{2}\right) \mathrm{e}^{\mathrm{j} k_{\mathrm{II} y}\left(L_{1}+L_{2}\right)}, \\
& \mathbf{A}(17,11)=-\omega(1-h+h g)\left(k_{x} / k_{r}^{2}\right) \mathrm{e}^{-\mathrm{j} k_{r y}\left(L_{1}+L_{2}\right)}, \\
& \mathbf{A}(17,12)=-\omega(1-h+h g)\left(k_{x} / k_{r}^{2}\right) \mathrm{e}^{\mathrm{j} k_{r y}\left(L_{1}+L_{2}\right)}, \\
& \mathbf{A}(17,13)=-\mathrm{j} k_{3 y} \mathrm{e}^{-\mathrm{j} k_{3 y}\left(L_{1}+L_{2}\right)}, \quad \mathbf{A}(17,14)=\mathrm{j} k_{3 y} \mathrm{e}^{\mathrm{j} k_{3 y}\left(L_{1}+L_{2}\right)} ; \\
& \mathbf{A}(18,7)=\left(2 k_{x} k_{\mathrm{I} y} / k_{\mathrm{I}}^{2}\right) \mathrm{e}^{-\mathrm{j} k_{\mathrm{I} y}\left(L_{1}+L_{2}\right)}, \quad \mathbf{A}(18,8)=-\left(2 k_{x} k_{\mathrm{I} y} / k_{\mathrm{I}}^{2}\right) \mathrm{e}^{\mathrm{j} k_{\mathrm{I} y}\left(L_{1}+L_{2}\right)}, \\
& \mathbf{A}(18,9)=\left(2 k_{x} k_{\mathrm{II} y} / k_{\mathrm{II}}^{2}\right) \mathrm{e}^{-\mathrm{j} k_{\mathrm{II} y}\left(L_{1}+L_{2}\right)}, \quad \mathbf{A}(18,10)=-\left(2 k_{x} k_{\mathrm{II} y} / k_{\mathrm{II}}^{2}\right) \mathrm{e}^{\mathrm{j} k_{\mathrm{II} y}\left(L_{1}+L_{2}\right)}, \\
& \mathbf{A}(18,11)=\left[\left(k_{x}^{2}-k_{r y}^{2}\right) / k_{r}^{2}\right] \mathrm{e}^{-\mathrm{j} k_{r y}\left(L_{1}+L_{2}\right)}, \quad \mathbf{A}(18,12)=\left[\left(k_{x}^{2}-k_{r y}^{2}\right) / k_{r}^{2}\right] \mathrm{e}^{\mathrm{j} k_{r y}\left(L_{1}+L_{2}\right)} ; \\
& \mathbf{A}(19,13)=\mathrm{j} k_{y} \mathrm{e}^{-\mathrm{j} k_{y}\left(L_{1}+L_{2}+\delta\right)}, \quad \mathbf{A}(19,14)=-\mathrm{j} k_{y} \mathrm{e}^{\mathrm{j} k_{y}\left(L_{1}+L_{2}+\delta\right)}, \quad \mathbf{A}(19,17)=-\mathrm{j} \omega ; \\
& \mathbf{A}(20,17)=-\mathrm{j} \omega, \quad \mathbf{A}(20,21)=\mathrm{j} k_{y} \mathrm{e}^{-\mathrm{j} k_{y}\left(L_{1}+L_{2}+\delta\right)} ; \\
& \mathbf{A}(21,13)=\mathrm{j} \omega \rho_{0} \mathrm{e}^{-\mathrm{j} k_{y}\left(L_{1}+L_{2}+\delta\right)}, \quad \mathbf{A}(21,14)=\mathrm{j} \omega \rho_{0} \mathrm{e}^{\mathrm{j} k_{y}\left(L_{1}+L_{2}+\delta\right)}, \\
& \mathbf{A}(21,17)=\omega^{2} m_{s 3}-D_{3} k_{x}^{4}, \quad \mathbf{A}(21,21)=-\mathrm{j} \omega \rho_{0} \mathrm{e}^{-\mathrm{j} k_{y}\left(L_{1}+L_{2}+\delta\right)} .
\end{aligned}
$$

Note that the above elements of $\mathbf{A}$ are for two foam layers with identical poroelastic material properties. If the two foam layers are different including the case of different interstitial air properties, the relevant coefficients must be calculated separately using material parameters of each individual foam layer. 


\section{References}

[1] F. X. Xin, T. J. Lu, C. Q. Chen, Vibroacoustic behavior of clamp mounted double-panel partition with enclosure air cavity, Journal of the Acoustical Society of America 124 (6) (2008) 3604-3612.

[2] F. X. Xin, T. J. Lu, External mean flow influence on noise transmission through double-leaf aeroelastic plates, AIAA Journal 47 (8) (2009) $1939-1951$.

[3] F. X. Xin, T. J. Lu, Analytical modelling of sound transmission across finite aeroelastic panels in convected fluids, Journal of the Acoustical Society of America 128 (3) (2010) 1097-1107.

[4] F. X. Xin, T. J. Lu, C. Chen, Sound transmission through lightweight all-metallic sandwich panels with corrugated cores, Advanced Materials Research 47-50 (2008) 57-60.

[5] F. X. Xin, T. J. Lu, Effects of core topology on sound insulation performance of lightweight all-metallic sandwich panels, Materials and Manufacturing Processes 26 (9) (2011) 1213-1221.

[6] F. X. Xin, T. J. Lu, Sound radiation of orthogonally rib-stiffened sandwich structures with cavity absorption, Composites Science and Technology 70 (15) (2009) 2198-2206.

[7] F. X. Xin, T. J. Lu, Analytical modelling of fluid loaded orthogonally rib-stiffened sandwich structures: Sound transmission, Journal of Mechanics and Physics of Solids 58 (9) (2010) 1374-1396. 
[8] F. X. Xin, T. J. Lu, Transmission loss of orthogonally rib-stiffened double-panel structures with cavity absorption, Journal of the Acoustical Society of America 129 (4) (2011) 1919-1934.

[9] L. L. Beranek, G. A. Work, Sound transmission through multiple structures containing flexible blankets, Journal of the Acoustical Society of America 21 (4) (1949) 419-428.

[10] W. Lauriks, P. Mees, J. F. Allard, The acoustic transmission through layered systems, Journal of Sound and Vibration 155 (1) (1992) 125-132.

[11] J. S. Bolton, E. R. Green, Normal incidence sound transmission through double-panel systems lined with relatively stiff, partially reticulated polyurethane foam, Applied Acoustics 39 (1993) 23-51.

[12] A. London, Transmission of reverberate sound through double walls, Journal of the Acoustical Society of America 22 (2) (1950) 270-279.

[13] K. A. Mulholland, A. J. Price, H. D. Parbrook, Transmission of multiple panels in a random incidence field, Journal of the Acoustical Society of America 43 (6) (1968) 1432-1435.

[14] J. S. Bolton, N. M. Shiau, Y. J. Kang, Sound transmission through multi-panel structures lined with elastic porous materials, Journal of Sound and Vibration 191 (3) (1996) 317-347.

[15] M. A. Biot, Theory of propagation of elastic waves in a fluid-saturated porous solid I. Low-frequency range. II. Higher frequency range, Journal of the Acoustical Society of America 28 (2) (1956) 168-191. 
[16] R. Panneton, N. Atalla, Numerical prediction of sound transmission through finite multilayer systems with poroelastic materials, Journal of the Acoustical Society of America 100 (1) (1996) 346-354.

[17] F. C. Sgard, N. Atalla, J. Nicolas, A numerical model for the low frequency diffuse field sound transmission loss of double-wall sound barriers with elastic porous linings, Journal of the Acoustical Society of America 108 (6) (2000) 2865-2872.

[18] O. Tanneau, J. B. Casimir, P. Lamary, Optimization of multilayered panels with poroelastic components for an acoustical transmission objective, Journal of the Acoustical Society of America 120 (3) (2006) $1227-1238$.

[19] J. S. Lee, E. I. Kim, Y. Y. Kim, J. S. Kim, Y. J. Kang, Optimal poroelastic layer sequencing for sound transmission loss maximization by topology optimization method, Journal of the Acoustical Society of America 122 (4) (2007) 2097-2106.

[20] J. Zhou, A. Bhaskar, X. Zhang, Optimization for sound transmission through a double-wall panel, Applied Acoustics 74 (12) (2013) $1422-1428$.

[21] J. Zhou, A. Bhaskar, X. Zhang, Sound transmission through a double-panel construction lined with poroelastic material in the presence of mean flow, Journal of Sound and Vibration 332 (16) (2013) 3724-3734.

[22] J. F. Allard, N. Atalla, Propagation of sound in porous media: modelling 
sound absorbing materials, 2nd Edition, John Wiley \& Sons Ltd., West Sussex, UK, 2009.

[23] D. A. Bies, C. H. Hansen, Engeneering Noise Control: Theory and Practice, 4th Edition, Spon Press, Abingdon, UK, 2009.

[24] A. J. B. Tadeu, D. M. R. Mateus, Sound transmission through single, double and triple glazing. Experimental evaluation, Applied Acoustics 62 (3) (2001) 307-325.

[25] A. Brekke, Calculation methods for the transmission loss of single, double and triple partitions, Applied Acoustics 14 (3) (1981) 225-240.

[26] F. X. Xin, T. J. Lu, Analytical modeling of sound transmission through clamped triple-panel partition separated by enclosed air cavities, European Journal of Mechanics A/Solids 30 (6) (2011) 770-782.

[27] B. H. Sharp, A study of techniques to increase of the sound insulation of building elements, PB 222 829, U. S. Department of Commerce, National Technical Information Service (NTIS) (1973).

[28] R. E. Jones, Sound insulation evaluation of high-performance wood-frame party partitions under laboratory and field conditions, Research Paper FPL 309, U.S. Department of Agriculture, Madison, Wisconsin (1970).

[29] B. H. Sharp, Prediction methods for the sound reduction of building elements, Noise Control Engineering 11 (2) (1978) 53-63. 
[30] J. D. Quirt, Sound transmission through windows II. double and triple glazing, Journal of the Acoustical Society of America 74 (2) (1983) $534-542$.

[31] A. D. Pierce, Acoustics: An Introduction to Its Physical Principles and Applications, McGraw-Hill Book Co., New York, 1981.

[32] K. Attenborough, Acoustical characteristics of porous materials, Physics Reports 82 (1982) 179-227.

[33] J. H. Lee, J. Kim, H. J. Kim, Simplified method to solve sound transmission through structures lined with elastic porous material, Journal of the Acoustical Society of America 110 (5) (2001) 2282-2294.

[34] J. S. Bolton, Cepstral techniques in the measurement of acoustic reflection coefficients, with application to the determination of acoustic properties of elastic porous materials, Ph.D. thesis, Institute of Sound and Vibration Research, University of Southampton, Southampton, UK (1984).

[35] J. F. Allard, C. Depollier, W. Lauriks, Measurement and prediction of surface impedance at oblique incidence of a plastic foam of high flow resistivity, Journal of Sound and Vibration 132 (1989) 51-60.

[36] L. Cremer, M. Heckl, E. E. Ungar, Structure-Borne Sound, Springer-Verlag, Berlin, 1973.

[37] K. A. Mulholland, H. D. Parbrook, A. Cummings, The transmission loss of double panels, Journal of Sound and Vibration 6 (1967) 324-334. 
[38] J. Wang, T. J. Lu, J. Woodhouse, R. S. Langley, J. Evans, Sound transmission through lightweight double-leaf partitions: theoretical modelling, Journal of Sound and Vibration 286 (2005) 817-847.

[39] G. F. C. Rogers, Y. R. Mayhew, Thermodynamic and Transport Properties of Fluids, 5th Edition, Blackwell Publishing Ltd., Oxford, 1995.

[40] Y. Y. Tang, R. J. Silcox, Sound transmission through two concentric cylindrical sandwich shells, in: Proceedings of 14th International Modal Analysis Conference, Detroit, 1996.

[41] B. Liu, L. Feng, A. Nilson, Influence of overpressure on sound transmission through curved panels, Journal of Sound and Vibration 302 (4) (2007) 760-776. 


\section{List of figure captions}

Figure 1: Schematic of a plane wave transmitting through a triple-panel structure.

Figure 2: The three configurations of a double-panel structure considered in Ref. [14].

Figure 3: Six typical configurations of a triple-panel structure considered in this study.

Figure 4: Comparison of transmission loss for a triple-panel structure without porous lining between current predictions and a previous model by Xin and $\mathrm{Lu}[26]$. Panel and air gap dimensions are $\delta_{1}=\delta_{2}=0.1 \mathrm{~m}$ and $h_{p 1}=h_{p 2}=h_{p 3}=0.002 \mathrm{~m}$.

Figure 5: Comparison of double-panel transmission loss between current predictions and the predicted and experimental results of Bolton et al. [14]. (a) BB, (b) BU, (c) UU.

Figure 6: Transmission loss of a double- (AA) and a triple-panel (AAAA) structures without porous linings.

Figure 7: Transmission loss of six typical triple-panel structures.

Figure 8: Comparison of transmission loss between typical double- and triple-panel structures.

Figure 9: Transmission loss of the (a) BB, (b) BU, (c) BBBB and (d) BUUB configurations with varying foam thickness.

Figure 10: Transmission loss of the (a) BBBB and (b) BUUB configurations with different varying thicknesses of the two foam layers.

Figure 11: Transmission loss of the (a) BU, (b) UU, (c) BBBU and (d) BUUB configurations with different air gap pressures. 